\title{
Lectures on the dynamical Yang-Baxter equations
}

\author{
Pavel Etingof and Olivier Schiffmann
}

\section{Introduction}

This paper arose from a minicourse given by the first author at MIT in the Spring of 1999, when the second author extended and improved his lecture notes of this minicourse. It contains a systematic and elementary introduction to a new area of the theory of quantum groups - the theory of the classical and quantum dynamical Yang-Baxter equations.

The quantum dynamical Yang-Baxter equation is a generalization of the ordinary quantum Yang-Baxter equation. It first appeared in physical literature in the work of Gervais and Neveu $[\overline{G N}$, and was first considered from a mathematical viewpoint by Felder $[\mathrm{F}]$, who attached to every solution of this equation a quantum group, and an interesting system of difference equations, - the quantum Knizhnik-Zamolodchikov-Bernard (qKZB) equation. Felder also considered the classical analogue of the quantum dynamical Yang-Baxter equation - the classical dynamical Yang-Baxter equation. Since then, this theory was systematically developed in many papers, some of which are listed below. By now, the theory of the classical and quantum dynamical Yang-Baxter equations and their solutions has many applications, in particular to integrable systems and representation theory. To discuss this theory and some of its applications is the goal of this paper.

The structure of the paper is as follows.

In Section 2 we consider the exchange construction, which is a natural construction in classical representation theory that leads one to discover the quantum dynamical Yang-Baxter equation and interesting solutions of this equation (dynamical R-matrices). In this section we define the main objects of the paper - the fusion and exchange matrices for Lie algebras and quantum groups, and compute them for the Lie algebra $s l_{2}$ and quantum group $U_{q}\left(s l_{2}\right)$.

In Section 3 we define the quantum dynamical Yang-Baxter equation, and see that the exchange matrices are solutions of this equation. We also study the quasiclassical limit of the quantum dynamical Yang-Baxter equation - the classical dynamical Yang-Baxter equation. We conjecture that any solution of this equation can be quantized. We compute classical limits of exchange matrices, which provides interesting examples of solutions of the classical dynamical Yang-Baxter equation, which we call basic solutions.

In Section 4 we give a classification of solutions of the classical dynamical Yang-Baxter equation for simple Lie algebras defined on a Cartan subalgebra, satisfying the unitarity condition. The result is, roughly, that all such solutions can be obtained from the basic solutions. 
In Section 5 we discuss the geometric interpretation of solutions of the classical dynamical Yang-Baxter equation, which generalizes Drinfeld's geometric interpretation of solutions of the classical Yang-Baxter equation via Poisson-Lie groups. This interpretation is in terms of Poisson-Lie groupoids introduced by Weinstein.

In Section 6 we give a classification of solutions of the quantum dynamical Yang-Baxter equation for the vector representation of $g l_{N}$, satisfying the Hecke condition. As in the classical case, the result states that all such solutions can be obtained from the basic solutions which arise from the exchange construction.

In Section 7 we discuss the "noncommutative geometric" interpretation of solutions of the quantum dynamical Yang-Baxter equation, which generalizes the interpretation of solutions of the quantum Yang-Baxter equation via quantum groups. This interpretation is in terms of quantum groupoids (or, more precisely, $H$-Hopf algebroids).

In Section 8 we give a defining equation satisfied by the universal fusion matrix - the Arnaudon-Buffenoir-Ragoucy-Roche (ABRR) equation, and prove it in the Lie algebra case. We give applications of this equation to computing the quasiclassical limit of the fusion matrix, and to computation of the fusion matrix itself for $s l_{2}$.

In Section 9 we discuss the connection of solutions of the quantum dynamical Yang-Baxter equation to integrable systems and special functions, in particular to Macdonald's theory. Namely, we consider weighted traces of intertwining operators between representations of quantum groups, and give difference equations for them which in a special case reduce to Macdonald-Ruijsenaars difference equations.

Appendix A contains the classification of solutions of the classical dynamical Yang-Baxter equation for simple Lie algebras defined on subspaces of the Cartan subalgebra.

Appendix B contains a proof of the ABRR equation in the quantum case.

At the end we review some of the existing literature that is relevant to the theory of the dynamical Yang-Baxter equations.

To keep these lectures within bounds, we do not discuss dynamical YangBaxter equations with spectral parameter. These equations are related to affine Lie algebras and quantum affine algebras just like the equations without spectral parameter are related to finite dimensional Lie algebras and quantum groups. Most of the definitions and results of these lectures can be carried over to this case, which gives rise to a more interesting but also more complicated theory than the theory described here. A serious discussion of this theory would require a separate course of lectures.

Acknowledgements. We thank the participants of the minicourse at MIT and of the "Quantum groups" conference in Durham (July 1999) for interesting remarks and discussions. We are grateful to IHES and Harvard University for hospitality. The work of P.E. was partially supported by the NSF grant DMS9700477, and was partly done while he was employed by the Clay Mathematics Institute as a CMI Prize Fellow. 


\section{Intertwining operators, fusion and exchange matrices.}

2.1. The exchange construction. We start by giving a simple and natural construction in classical representation theory which leads to discovery of the quantum dynamical Yang-Baxter equation.

Let $\mathfrak{g}$ be a simple complex Lie algebra, $\mathfrak{h} \subset \mathfrak{g}$ a Cartan subalgebra and $\Delta \subset \mathfrak{h}^{*}$ the associated root system. Let $\Pi$ be a set of simple roots, $\Delta^{+} \subset \Delta$ the associated system of positive roots. Let $\mathfrak{g}=\mathfrak{n}_{-} \oplus \mathfrak{h} \oplus \mathfrak{n}_{+}$be the corresponding polarization of $\mathfrak{g}$ and let $\mathfrak{g}_{\alpha}$ be the root subspaces of $\mathfrak{g}$. Let $\langle$,$\rangle be the nonde-$ generate invariant symmetric form on $\mathfrak{g}$ normalized by the condition $\langle\alpha, \alpha\rangle=2$ for long roots. Finally, for each $\alpha \in \Delta$, choose some $e_{\alpha} \in \mathfrak{g}_{\alpha}$ in such a way that $\left\langle e_{\alpha}, e_{-\alpha}\right\rangle=1$.

For $\lambda \in \mathfrak{h}^{*}$, let $\mathbb{C}_{\lambda}$ be the one-dimensional $\left(\mathfrak{h} \oplus \mathfrak{n}_{+}\right)$-module such that $\mathbb{C}_{\lambda}=\mathbb{C} x_{\lambda}$ with $h \cdot x_{\lambda}=\lambda(h) x_{\lambda}$ for $h \in \mathfrak{h}$ and $\mathfrak{n}_{+} \cdot x_{\lambda}=0$. The Verma module of highest weight $\lambda$ is the induced module

$$
M_{\lambda}=\operatorname{Ind}_{\mathfrak{h} \oplus \mathfrak{n}_{+}}^{\mathfrak{g}} \mathbb{C}_{\lambda} .
$$

Notice that $M_{\lambda}$ is a free $U\left(\mathfrak{n}_{-}\right)$-module and can be identified with $U\left(\mathfrak{n}_{-}\right)$as a linear space by the map $U\left(\mathfrak{n}_{-}\right) \stackrel{\sim}{\rightarrow} M_{\lambda}, u \mapsto u . x_{\lambda}$.

Define a partial order on $\mathfrak{h}^{*}$ by putting $\mu<\nu$ if there exist $\alpha_{1}, \ldots \alpha_{r} \in \Delta^{+}$, $r>0$, such that $\nu=\mu+\alpha_{1}+\ldots+\alpha_{r}$. Let $M_{\lambda}=\bigoplus_{\mu \leq \lambda} M_{\lambda}[\mu]$ denote the decomposition of $M_{\lambda}$ into weight subspaces.

The following proposition is standard.

Proposition 2.1. The module $M_{\lambda}$ is irreducible for generic values of $\lambda$.

Define also the dual Verma module $M_{\lambda}^{*}$ to be the graded dual vector space $\bigoplus_{\mu} M_{\lambda}[\mu]^{*}$ equipped with the following $\mathfrak{g}$-action:

$$
(a . u)(v)=-u(a . v) \forall a \in \mathfrak{g}, u \in M_{\lambda}^{*}, v \in M_{\lambda} .
$$

Let $x_{\lambda}^{*}$ be the lowest weight vector of $M_{\lambda}^{*}$ satisfying $\left\langle x_{\lambda}, x_{\lambda}^{*}\right\rangle=1$.

Now let $V$ be a finite-dimensional $\mathfrak{g}$-module. Let $V=\bigoplus_{\nu \in \mathfrak{h}^{*}} V[\nu]$ be its decomposition into weight subspaces. Let $\lambda, \mu \in \mathfrak{h}^{*}$ and let us consider $\mathfrak{g}$-module intertwining operators

$$
\Phi: M_{\lambda} \rightarrow M_{\mu} \otimes V .
$$

If $\Phi$ is such an intertwining operator, define its "expectation value" by

$$
\langle\Phi\rangle=\left\langle\Phi . x_{\lambda}, x_{\mu}^{*}\right\rangle \in V[\lambda-\mu] .
$$

Remark. This definition is similar to the notion of expectation value in quantum field theory.

Proposition 2.2. Let $M_{\mu}$ be irreducible. Then the map

$$
\operatorname{Hom}_{\mathfrak{g}}\left(M_{\lambda}, M_{\mu} \otimes V\right) \rightarrow V[\lambda-\mu], \Phi \mapsto\langle\Phi\rangle
$$

is an isomorphism. 
Proof. By Frobenius reciprocity, we have

$$
\operatorname{Hom}_{\mathfrak{g}}\left(M_{\lambda}, M_{\mu} \otimes V\right)=\operatorname{Hom}_{\mathfrak{h} \oplus \mathfrak{n}_{+}}\left(\mathbb{C}_{\lambda}, M_{\mu} \otimes V\right)=\operatorname{Hom}_{\mathfrak{h} \oplus \mathfrak{n}_{+}}\left(\mathbb{C}_{\lambda} \otimes M_{\mu}^{*}, V\right) .
$$

Moreover, since $M_{\mu}$ is irreducible, we have $M_{\mu}^{*}=\operatorname{Ind}_{\mathfrak{h}}^{\mathfrak{h} \oplus \mathfrak{n}_{+}} \mathbb{C}_{-\mu}$ as an $\mathfrak{h} \oplus \mathfrak{n}_{+^{-}}$ module. In particular,

$$
\operatorname{Hom}_{\mathfrak{h} \oplus \mathfrak{n}_{+}}\left(\mathbb{C}_{\lambda} \otimes M_{\mu}^{*}, V\right)=\operatorname{Hom}_{\mathfrak{h}}\left(\mathbb{C}_{\lambda} \otimes \mathbb{C}_{-\mu}, V\right)=V[\lambda-\mu] .
$$

This proposition can be reformulated as follows: for any $v \in V[\lambda-\mu]$ there exists a unique intertwining operator $\Phi_{\lambda}^{v}: M_{\lambda} \rightarrow M_{\mu} \otimes V$ such that

$$
\Phi_{\lambda}^{v}\left(x_{\lambda}\right) \in x_{\mu} \otimes v+\bigoplus_{\nu<\mu} M_{\mu}[\nu] \otimes V .
$$

Notice that $\Phi_{\lambda}^{v}$ (for fixed $v$ ) is defined only for generic values of $\lambda$. Identifying the Verma modules $M_{\lambda}$ and $M_{\mu}$ with $U\left(\mathfrak{n}_{+}\right)$, we can view $\Phi_{\lambda}^{v}$ as a linear map $U\left(\mathfrak{n}_{+}\right) \rightarrow U\left(\mathfrak{n}_{+}\right) \otimes V$. It is easy to see that the coefficients of this map (in any basis) are rational functions of $\lambda$.

We would now like to consider the "algebra" of such intertwining operators. Let us denote by $\operatorname{wt}(u) \in \mathfrak{h}^{*}$ the weight of any homogeneous vector $u$ in a $\mathfrak{g}$ module. Let $V, W$ be two finite-dimensional $\mathfrak{g}$-modules, and let $v \in V, w \in W$ be two homogeneous vectors. Let $\lambda \in \mathfrak{h}^{*}$ and consider the composition

$$
\Phi_{\lambda}^{w, v}: M_{\lambda} \stackrel{\Phi_{\lambda}^{v}}{\longrightarrow} M_{\lambda-\mathrm{wt}(v)} \otimes V \stackrel{\Phi_{\lambda-\mathrm{wt}(v)}^{w}}{\longrightarrow} M_{\lambda-\mathrm{wt}(v)-\mathrm{wt}(w)} \otimes W \otimes V .
$$

(Here and below we abuse notations and write $\Phi$ instead of $\Phi \otimes 1$ ). Then $\Phi_{\lambda}^{w, v} \in \operatorname{Hom}_{\mathfrak{g}}\left(M_{\lambda}, M_{\lambda-\mathrm{wt}(v)-\mathrm{wt}(w)} \otimes W \otimes V\right)$. Hence by Proposition 1.2, for generic $\lambda$ there exists a unique element $u \in V \otimes W[\operatorname{wt}(v)+\operatorname{wt}(w)]$ such that $\Phi_{\lambda}^{u}=\Phi_{\lambda}^{w, v}$. It is clear that the assignment $(v, w) \mapsto u$ is bilinear, and defines an $\mathfrak{h}$-linear map

$$
\begin{aligned}
J_{W V}(\lambda): W \otimes V & \rightarrow W \otimes V, \\
w \otimes v & \mapsto\left\langle\Phi_{\lambda}^{w, v}\right\rangle
\end{aligned}
$$

Definition. We call the operator $J_{W V}(\lambda)$ the fusion matrix of $V$ and $W$.

We will now list some fundamental properties of fusion matrices. First let us introduce an important piece of notation to be used throughout this text. If $A_{1}, \ldots A_{r}$ are semisimple $\mathfrak{h}$-modules and $F(\lambda): A_{1} \otimes \ldots \otimes A_{r} \rightarrow A_{1} \otimes \ldots \otimes A_{r}$ is a linear operator depending on $\lambda \in \mathfrak{h}^{*}$ then, for any homogeneous $a_{1}, \ldots a_{r}$ we set

$$
F\left(\lambda-h^{(i)}\right)\left(a_{1} \otimes \ldots \otimes a_{r}\right):=F\left(\lambda-\operatorname{wt}\left(a_{i}\right)\right)\left(a_{1} \otimes \ldots \otimes a_{r}\right) .
$$

Proposition 2.3. Let $V, W$ be finite-dimensional $\mathfrak{g}$-modules. Then 
1. $J_{W V}(\lambda)$ is a rational function of $\lambda$.

2. $J_{W V}(\lambda)$ is strictly lower triangular, i.e. $J=1+N$ where

$$
N(W[\nu] \otimes V[\mu]) \subset \bigoplus_{\tau<\nu, \mu<\sigma} W[\tau] \otimes V[\sigma] .
$$

In particular, $J_{W V}(\lambda)$ is invertible.

3. Let $U, V, W$ be finite-dimensional $\mathfrak{g}$-modules. Then the fusion matrices satisfy the following dynamical 2-cocycle condition:

$$
J_{U \otimes W, V}(\lambda)\left(J_{U W}\left(\lambda-h^{(3)}\right) \otimes 1\right)=J_{U, W \otimes V}(\lambda)\left(1 \otimes J_{W V}(\lambda)\right) .
$$

on $U \otimes V \otimes W$.

Proof. Statements 1. and 2. follow from the definitions and from the fact that the intertwining operators $\Phi_{\lambda}^{v}$ are rational functions of $\lambda$. To prove statement 3., let $u \in U, v \in V, w \in W$ be homogeneous elements and consider the composition

$$
\begin{aligned}
& M_{\lambda} \stackrel{\Phi_{\lambda}^{v}}{\longrightarrow} M_{\lambda-\mathrm{wt}(v)} \otimes V \stackrel{\Phi_{\lambda-\mathrm{wt}(v)}^{w}}{\longrightarrow} M_{\lambda-\mathrm{wt}(v)-\mathrm{wt}(w)} \otimes W \otimes V \\
& \Phi_{\lambda-\mathrm{wt}(v)-\mathrm{wt}(w)}^{u} M_{\lambda-\mathrm{wt}(u)-\mathrm{wt}(v)-\mathrm{wt}(w)} \otimes U \otimes W \otimes V .
\end{aligned}
$$

The dynamical 2-cocycle condition follows from the associativity relation

$$
\Phi_{\lambda-\operatorname{wt}(v)-\operatorname{wt}(w)}^{u} \circ\left(\Phi_{\lambda-\operatorname{wt}(v)}^{w} \circ \Phi_{\lambda}^{v}\right)=\left(\Phi_{\lambda-\operatorname{wt}(v)-\operatorname{wt}(w)}^{u} \circ \Phi_{\lambda-\operatorname{wt}(v)}^{w}\right) \circ \Phi_{\lambda}^{v}
$$

and from the definition of the fusion matrices.

The fusion matrices can be viewed as the structure constants for multiplication in the "algebra" of intertwining operators. We now turn to the structure constants for "commutation relations". Let $V, W$ be two finite-dimensional $\mathfrak{g}$ modules. Let us define

$$
R_{V W}(\lambda)=J_{V W}(\lambda)^{-1} J_{W V}^{21}(\lambda) \in \operatorname{Hom}_{\mathfrak{h}}(V \otimes W, V \otimes W),
$$

where $J^{21}=P J P$ with $P(x \otimes y)=y \otimes x$. The above definition can be rephrased in terms of intertwining operators as follows: $R_{V W}(\lambda)(v \otimes w)=\sum_{i} v_{i} \otimes w_{i}$ where $\Phi_{\lambda}^{w, v}=P \sum_{i} \Phi_{\lambda}^{v_{i}, w_{i}}$

Definition. The operator $R_{V W}(\lambda)$ is called the exchange matrix of $V$ and $W$.

Proposition 2.4. Let $U, V, W$ be three finite-dimensional $\mathfrak{g}$-modules. Then the exchange matrices satisfy the following relation

$$
R_{V W}\left(\lambda-h^{(3)}\right) R_{V U}(\lambda) R_{W U}\left(\lambda-h^{(1)}\right)=R_{W U}(\lambda) R_{V U}\left(\lambda-h^{(2)}\right) R_{V W}(\lambda)
$$

in the algebra $\operatorname{Hom}_{\mathfrak{h}}(V \otimes W \otimes U, V \otimes W \otimes U)$. 
Proof. Let $u \in U, v \in V, w \in W$ be homogeneous elements and, as in Proposition 2.3, consider the composition $\Phi_{\lambda}^{u, w, v}=\Phi_{\lambda-\operatorname{wt}(v)-\operatorname{wt}(w)}^{u} \circ \Phi_{\lambda-\operatorname{wt}(v)}^{w} \circ \Phi_{\lambda}^{v}$. The proof of relation 2.1) is obtained by rewriting $\Phi_{\lambda}^{u, w, v}$ as $\sum \sigma \Phi_{\lambda}^{v_{i}, w_{i}, u_{i}}$ where $\sigma: U \otimes W \otimes V \rightarrow V \otimes W \otimes U, x \otimes y \otimes z \mapsto z \otimes y \otimes x$, using exchange matrices in two different ways according to the following hexagon

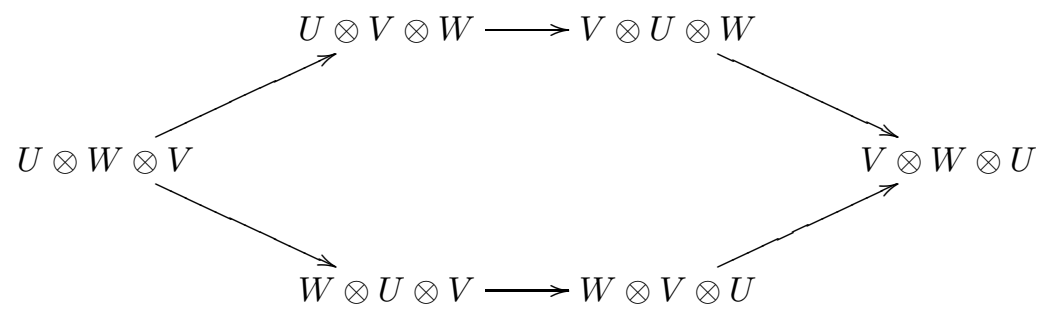

Remark. One can also deduce this proposition from Part 3. of Proposition 2.3. Namely, one can show that if $J(\lambda)$ is any element of the completion of $U(\mathfrak{g}) \otimes U(\mathfrak{g})$ which satisfies the dynamical 2-cocycle condition (where $J_{V W}(\lambda)$ denotes the projection of $J(\lambda)$ to the product $V \otimes W$ of finite dimensional modules $V, W$ ) then the element $R(\lambda)=J(\lambda)^{-1} J^{21}(\lambda)$ satisfies the quantum dynamical Yang-Baxter equation.

Example 1. Let us evaluate the fusion and exchange matrices in the simplest example. Namely, take $\mathfrak{g}=\mathfrak{s l} \mathfrak{l}_{2}=\mathbb{C} e \oplus \mathbb{C} h \oplus \mathbb{C} f$ and $V=\mathbb{C}^{2}=\mathbb{C} v_{+} \oplus \mathbb{C} v_{-}$with

$$
h . v_{ \pm}= \pm v_{ \pm}, \quad e . v_{-}=v_{+}, \quad e . v_{+}=0, \quad f . v_{-}=0, \quad f \cdot v_{+}=v_{-} .
$$

Let us compute the fusion matrix $J_{V V}(\lambda)$. By the triangularity property of $J_{V V}(\lambda)$, we have

$$
J_{V V}(\lambda)\left(v_{ \pm} \otimes v_{ \pm}\right)=v_{ \pm} \otimes v_{ \pm}, \quad J_{V V}(\lambda)\left(v_{-} \otimes v_{+}\right)=v_{-} \otimes v_{+},
$$

so it remains to compute $J_{V V}(\lambda)\left(v_{-} \otimes v_{+}\right)$. Consider the intertwiner $\Phi_{\lambda}^{v_{-}}$: $M_{\lambda} \rightarrow M_{\lambda+1} \otimes V$. By definition, $\Phi_{\lambda}^{v_{-}}\left(x_{\lambda}\right)=x_{\lambda+1} \otimes v_{-}+y(\lambda) f x_{\lambda+1} \otimes v_{+}$. To determine the function $y(\lambda)$, we use the intertwining property:

$$
\begin{aligned}
0=\Phi_{\lambda}^{v_{-}}\left(e x_{\lambda}\right)=(e \otimes 1+1 \otimes e) \Phi_{\lambda}^{v_{-}}\left(x_{\lambda}\right) & =x_{\lambda+1} \otimes v_{+}+y(\lambda) e f x_{\lambda+1} \otimes v_{+} \\
& =x_{\lambda+1} \otimes v_{+}+y(\lambda)(h+f e) x_{\lambda+1} \otimes v_{+} \\
& =x_{\lambda+1} \otimes v_{+}+(\lambda+1) y(\lambda) x_{\lambda+1} \otimes v_{+}
\end{aligned}
$$

Hence $y(\lambda)=-\frac{1}{\lambda+1}$. A similar computation shows that $\Phi_{\lambda+1}^{v_{+}}\left(x_{\lambda+1}\right)=x_{\lambda} \otimes v_{+}$. Thus

$\Phi_{\lambda}^{v_{+}, v_{-}}\left(x_{\lambda}\right)=\Phi_{\lambda+1}^{v_{+}} \Phi_{\lambda}^{v_{-}}\left(x_{\lambda}\right)=x_{\lambda} \otimes\left(v_{+} \otimes v_{-}-\frac{1}{\lambda+1} v_{-} \otimes v_{+}\right)+$lower weight terms.

Therefore $J_{V V}(\lambda)\left(v_{+} \otimes v_{-}\right)=v_{+} \otimes v_{-}-\frac{1}{\lambda+1} v_{-} \otimes v_{+}$, and

$$
J_{V V}(\lambda)=\left(\begin{array}{cccc}
1 & 0 & 0 & 0 \\
0 & 1 & 0 & 0 \\
0 & -\frac{1}{\lambda+1} & 1 & 0 \\
0 & 0 & 0 & 1
\end{array}\right)
$$


The exchange matrix is now easily computed. In the basis $\left(v_{+} \otimes v_{+}, v_{+} \otimes v_{-}\right.$, $\left.v_{-} \otimes v_{+}, v_{-} \otimes v_{-}\right)$it is given by

$$
R_{V V}(\lambda)=\left(\begin{array}{cccc}
1 & 0 & 0 & 0 \\
0 & 1 & -\frac{1}{\lambda+1} & 0 \\
0 & \frac{1}{\lambda+1} & 1-\frac{1}{(\lambda+1)^{2}} & 0 \\
0 & 0 & 0 & 1
\end{array}\right)
$$

2.2. Generalization to quantum groups. The construction of intertwining operators, fusion and exchange matrices admit natural quantum analogues. Let $U_{q}(\mathfrak{g})$ be the quantum universal enveloping algebra associated to $\mathfrak{g}$, as defined in CP. Chapter 6., and for each $\lambda \in \mathfrak{h}^{*}$, let $M_{\lambda}$ be the Verma module of highest weight $\lambda$. Then Proposition 2.2 and the definition of the fusion matrices $J_{W, V}(\lambda)$ are identical to the classical case. In this situation, Proposition 2.3, parts 2., 3 . hold. However, the fusion matrices are no longer rational functions of $\lambda$, but rather trigonometric functions (i.e rational functions of $q^{<\lambda, \alpha>}, \alpha \in \Delta$ ).

Let $\mathcal{R} \in U_{q}(\mathfrak{g}) \hat{\otimes} U_{q}(\mathfrak{g})$ be the universal R-matrix of $U_{q}(\mathfrak{g})$. Let $V, W$ be two finite-dimensional $U_{q}(\mathfrak{g})$-modules. The exchange matrix is defined as

$$
R_{V W}(\lambda)=J_{V W}^{-1}(\lambda) \mathcal{R}_{V W}^{21} J_{W, V}^{21}(\lambda)
$$

where $\mathcal{R}_{V W}^{21}$ is the evaluation of $\mathcal{R}^{21}$ on $V \otimes W$.

In terms of intertwining operators, the exchange matrix has the following interpretation. Recall that if $V$ and $W$ are any two $U_{q}(\mathfrak{g})$-modules then $P \mathcal{R}_{V W}$ : $V \otimes W \rightarrow W \otimes V$ is a $U_{q}(\mathfrak{g})$-intertwiner. Then $R_{V W}(\lambda)(v \otimes w)=\sum_{i} v_{i} \otimes w_{i}$ where $P \mathcal{R}_{W V} \Phi_{\lambda}^{w, v}=\sum_{i} \Phi_{\lambda}^{v_{i}, w_{i}}$.

With this definition, Proposition 2.4 is satisfied. The quantum analogues of the fusion and exchange matrices in example 1 are

$$
\begin{gathered}
J_{V V}(\lambda)=\left(\begin{array}{cccc}
1 & 0 & 0 & 0 \\
0 & 1 & 0 & 0 \\
0 & \frac{q^{-1}-q}{q^{2(\lambda+1)}-1} & 1 & 0 \\
0 & 0 & 0 & 1
\end{array}\right), \\
R_{V V}(\lambda)=\left(\begin{array}{cccc}
q & 0 & 0 & 0 \\
0 & 1 & \frac{q^{-1}-q}{q^{2(\lambda+1)}-1} & 0 \\
0 & \frac{q^{-1}-q}{q^{-2(\lambda+1)}-1} & \frac{\left(q^{2(\lambda+1)}-q^{2}\right)\left(q^{2(\lambda+1)}-q^{-2}\right)}{\left(q^{2(\lambda+1)}-1\right)^{2}} & 0 \\
0 & 0 & 0 & q
\end{array}\right) .
\end{gathered}
$$

\section{The dynamical Yang-Baxter equations.}

3.1. Proposition 2.4 motivates the following definition. Let $\mathfrak{h}$ be a finitedimensional abelian Lie algebra and let $V$ be a semisimple $\mathfrak{h}$-module. Let us denote by $M$ the field of meromorphic functions on $\mathfrak{h}^{*}$. Let us equip $M$ with the trivial $\mathfrak{h}$-module structure.

Definition. Let $R: V \otimes V \otimes M \rightarrow V \otimes V \otimes M$ be an h-invariant and $M$ linear map. Then the quantum dynamical Yang-Baxter equation (QDYBE) is the following equation with respect to $R$ :

$$
R^{12}\left(\lambda-h^{(3)}\right) R^{13}(\lambda) R^{23}\left(\lambda-h^{(1)}\right)=R^{23}(\lambda) R^{13}\left(\lambda-h^{(2)}\right) R^{12}(\lambda) .
$$


A quantum dynamical R-matrix is an invertible solution of this equation.

It follows from Proposition 2.4 that for any simple complex Lie algebra $\mathfrak{g}$ and for any finite-dimensional $\mathfrak{g}$-module $V$, the exchange matrix $R_{V V}(\lambda)$ is a quantum dynamical R-matrix. The same is true if we replace the Lie algebra $\mathfrak{g}$ by the quantum group $U_{q}(\mathfrak{g})$.

Remarks. 1. The usual quantum Yang-Baxter equation is recovered from the quantum dynamical Yang-Baxter equation when $\mathfrak{h}=0$.

2. A constant solution of the quantum dynamical Yang-Baxter equation is the same thing as a solution of the ordinary quantum Yang-Baxter equation which is $\mathfrak{h}$-invariant.

3. In physical literature, the variable $\lambda$ is called a dynamical variable. This gave rise to the name "dynamical R-matrix".

Replacing $\lambda$ by $\frac{\lambda}{\gamma}$ in the QDYBE yields the following equation

$$
\tilde{R}^{12}\left(\lambda-\gamma h^{(3)}\right) \tilde{R}^{13}(\lambda) \tilde{R}^{23}\left(\lambda-\gamma h^{(1)}\right)=\tilde{R}^{23}(\lambda) \tilde{R}^{13}\left(\lambda-\gamma h^{(2)}\right) \tilde{R}^{12}(\lambda),
$$

which is called the quantum dynamical Yang-Baxter equation with step $\gamma$.

Proposition 3.1. Let $\mathfrak{h}$ be an abelian Lie algebra. Let $V$ be a finite-dimensional semisimple $\mathfrak{h}$-module and let $R: \mathfrak{h}^{*} \rightarrow \operatorname{End}_{\mathfrak{h}}(V \otimes V)[[\gamma]]$ be a series of meromorphic functions of the form $R=1-\gamma r+O\left(\gamma^{2}\right)$. If $R$ satisfies the quantum dynamical Yang-Baxter equation with step $\gamma$ then $r$ satisfies the following classical analogue of the quantum dynamical Yang-Baxter equation:

$$
\begin{aligned}
\sum_{i} & \left(x_{i}^{(1)} \frac{\partial r^{23}(\lambda)}{\partial x^{i}}-x_{i}^{(2)} \frac{\partial r^{13}(\lambda)}{\partial x^{i}}+x_{i}^{(3)} \frac{\partial r^{12}(\lambda)}{\partial x^{i}}\right)+ \\
& {\left[r^{12}(\lambda), r^{13}(\lambda)\right]+\left[r^{12}(\lambda), r^{23}(\lambda)\right]+\left[r^{13}(\lambda), r^{23}(\lambda)\right]=0 }
\end{aligned}
$$

where $\left(x_{i}\right)$ is a basis of $\mathfrak{h}$ and $\left(x^{i}\right)$ is the dual basis of $\mathfrak{h}^{*}$.

This leads to the following definition:

Definition. Let $\mathfrak{g}$ be a finite-dimensional Lie algebra and let $\mathfrak{h} \subset \mathfrak{g}$ be a Lie subalgebra. The classical dynamical Yang-Baxter equation (CDYBE) is equation (3.2) with respect to a holomorphic, $\mathfrak{h}$-invariant function $r: U \rightarrow \mathfrak{g} \otimes \mathfrak{g}$, where $U \subset \mathfrak{h}^{*}$ is an open region. A solution to this equation is called a classical dynamical r-matrix.

Remarks. 1. The ordinary classical Yang-Baxter equation is recovered from the classical dynamical Yang-Baxter equation when $\mathfrak{h}=0$.

2. A constant solution of the classical dynamical Yang-Baxter equation is the same thing as an $\mathfrak{h}$-invariant solution of the ordinary classical Yang-Baxter equation. 
We will now consider asymptotic behavior of fusion and exchange matrices, and obtain solutions to the CDYBE. Let $\mathfrak{g}$ be a simple complex Lie algebra. Let $V, W$ be two finite-dimensional $\mathfrak{g}$-modules and let $J_{V W}(\lambda)$ and $R_{V W}(\lambda)$ be the fusion and exchange matrices of $V$ and $W$.

Proposition 3.2 ([EV3]). 1. The function $J_{V W}\left(\frac{\lambda}{\gamma}\right)$ is regular at $\gamma=0$ for generic values of $\lambda$.

2. Set $J_{V W}\left(\frac{\lambda}{\gamma}\right)=1+\gamma j_{V W}(\lambda)+O\left(\gamma^{2}\right)$. Then $j_{V W}(\lambda)$ is the evaluation on $V \otimes W$ of the element

$$
j(\lambda)=-\sum_{\alpha>0} \frac{e_{-\alpha} \otimes e_{\alpha}}{\langle\alpha, \lambda\rangle} \in \mathfrak{n}_{-} \otimes \mathfrak{n}_{+}
$$

Corollary 3.1. We have $R_{V W}\left(\frac{\lambda}{\gamma}\right)=1-\gamma r(\lambda)_{\mid V \otimes W}+O\left(\gamma^{2}\right)$ where

$$
r(\lambda)=j(\lambda)-j^{21}(\lambda)=\sum_{\alpha>0} \frac{e_{\alpha} \otimes e_{-\alpha}-e_{-\alpha} \otimes e_{\alpha}}{\langle\alpha, \lambda\rangle} .
$$

A proof of Proposition 3.2, which is based on computing the asymptotics of intertwining operators at $\lambda \rightarrow \infty$, is given in EV3. Later we will give another proof of this Proposition.

It follows from Proposition 3.1 that $r(\lambda)$ in (3.3) is a classical dynamical r-matrix. Let us call it the basic rational dynamical r-matrix.

Proposition 3.2 and Corollary 3.1 have natural quantum analogues. Let $U_{q}(\mathfrak{g})$ be the quantum group associated to $\mathfrak{g}$ with quantum parameter $q=e^{-\varepsilon \gamma / 2}$ for some fixed $\varepsilon \in \mathbb{C}$ and formal parameter $\gamma$. Let $V, W$ be two finite-dimensional $U_{q}(\mathfrak{g})$-modules and let $R_{V W}(\lambda)$ be the exchange matrix. Set $\tilde{R}_{V W}(\lambda)=R_{V W}\left(\frac{\lambda}{\gamma}\right)$.

Proposition 3.3 ([EV3]). We have

$$
\tilde{R}_{V W}(\lambda)=1-\gamma r_{V W}^{\varepsilon}(\lambda)+O\left(\gamma^{2}\right)
$$

where $r_{V W}^{\varepsilon}: \mathfrak{h}^{*} \rightarrow \operatorname{End}_{\mathfrak{h}}(V \otimes W)$ is the evaluation on $V \otimes W$ of the following universal element:

$$
r^{\varepsilon}(\lambda)=\frac{\varepsilon}{2} \Omega+\sum_{\alpha>0} \frac{\varepsilon}{2} \operatorname{cotanh}\left(\frac{\varepsilon}{2}\langle\alpha, \lambda\rangle\right)\left(e_{\alpha} \otimes e_{-\alpha}-e_{-\alpha} \otimes e_{\alpha}\right) \in \mathfrak{g} \otimes \mathfrak{g}
$$

where $\Omega \in S^{2} \mathfrak{g}$ is the inverse element to the form (, ) (the Casimir element).

It follows from Proposition 3.1 that $r^{\varepsilon}(\lambda)$ is a solution of the CDYBE. Let us call it the basic trigonometric dynamical r-matrix.

3.2. Quantization and quasiclassical limit. Let $\mathfrak{h}$ be an abelian Lie algebra and let $V$ be a finite-dimensional semisimple $\mathfrak{h}$-module. Let $r: \mathfrak{h}^{*} \rightarrow$ $\operatorname{End}_{\mathfrak{h}}(V \otimes V)$ be a classical dynamical r-matrix. Suppose that $R: \mathfrak{h}^{*} \rightarrow$ $\operatorname{End}_{\mathfrak{h}}(V \otimes V)[[\gamma]]$ is of the form $R=1-\gamma r+O\left(\gamma^{2}\right)$ and satisfies the QDYBE.

Definition. $\quad R$ is called a quantization of $r$. Conversely, $r$ is called the quasiclassical limit of $R$. 
For instance, the exchange matrix $\tilde{R}_{V V}(\lambda)$ constructed from a Lie algebra $\mathfrak{g}$ is a quantization of the evaluation on $V \otimes V$ of the basic rational dynamical rmatrix. Similarly, exchange matrices constructed from quantum groups provide quantization of the basic trigonometric dynamical r-matrix.

Conjecture: Any classical dynamical $r$-matrix admits a quantization.

Notice that when $\mathfrak{h}=0$, this conjecture reduces to the conjecture of Drinfeld [Dr] about quantization of classical (non-dynamical) r-matrices, which was proved in [EK]. In the skew-symmetric case, the conjecture was recently proved in Xu2], using the theory of Fedosov quantization.

3.3. Unitarity conditions. Recall the following notions introduced by Drinfeld. A classical r-matrix $r \in \mathfrak{g} \otimes \mathfrak{g}$ is a quasitriangular structure on a Lie algebra $\mathfrak{g}$ if $r+r^{21} \in\left(S^{2} \mathfrak{g}\right)^{\mathfrak{g}}$. It is a triangular structure on $\mathfrak{g}$ if $r+r^{21}=0$.

This definition is natural in the theory of Lie bialgebras. Namely, a classical r-matrix $r \in \mathfrak{g} \otimes \mathfrak{g}$ defines a Lie bialgebra structure on $\mathfrak{g}$ by $\delta: \mathfrak{g} \rightarrow \Lambda^{2} \mathfrak{g}, x \mapsto$ $[1 \otimes x+x \otimes 1, r]$ if and only if $r+r^{21} \in\left(S^{2} \mathfrak{g}\right)^{\mathfrak{g}}$. In the case of a simple Lie algebra $\mathfrak{g}$ we have $\left(S^{2} \mathfrak{g}\right)^{\mathfrak{g}}=\mathbb{C} \Omega$, so that a classical r-matrix $r$ is quasitriangular if $r+r^{21}=\varepsilon \Omega$ for some $\varepsilon \in \mathbb{C}$, and it is triangular if moreover $\varepsilon=0$.

This leads one to make the following definition:

Definition. A classical dynamical r-matrix $r: \mathfrak{h}^{*} \rightarrow(\mathfrak{g} \otimes \mathfrak{g})^{\mathfrak{h}}$ has coupling constant $\varepsilon$ if

$$
r+r^{21}=\varepsilon \Omega .
$$

Equation (3.5) is called the unitarity condition. Notice that the basic rational dynamical r-matrix $r(\lambda)$ and the basic trigonometric dynamical r-matrix $r^{\varepsilon}(\lambda)$ have coupling constants 0 and $\varepsilon$ respectively.

\section{Classification of classical dynamical $r$-matrices.}

In this section, we give the classification of all dynamical r-matrices $r: \mathfrak{h}^{*} \rightarrow$ $\mathfrak{g} \otimes \mathfrak{g}$ which have coupling constant $\varepsilon \in \mathbb{C}$.

4.1. Gauge transformations. Consider the following operations on meromorphic maps $r: \mathfrak{h}^{*} \rightarrow(\mathfrak{g} \otimes \mathfrak{g})^{\mathfrak{h}}$.

1. $r(\lambda) \mapsto r(\lambda)+\sum_{i<j} C_{i j}(\lambda) x_{i} \wedge x_{j}$, where $\sum_{i, j} C_{i j}($ lambda $) d \lambda_{i} \wedge d \lambda_{j}$ is a closed meromorphic 2-form.

2. $r(\lambda) \mapsto r(\lambda-\nu)$, where $\nu \in \mathfrak{h}^{*}$.

3. $r(\lambda) \mapsto(A \otimes A) r\left(A^{*} \lambda\right)$, where $A \in W$, the Weyl group of $\mathfrak{g}$.

Lemma 4.1. Transformations 1-3 preserve the set of classical dynamical $r$ matrices. 
The proof is straightforward.

Two classical dynamical r-matrices which can be obtained one from the other by a sequence of such transformations will be called gauge-equivalent.

4.2. Classification of dynamical r-matrices with zero coupling constant. Let $\mathfrak{l} \supset \mathfrak{h}$ be a reductive Lie subalgebra of $\mathfrak{g}$. Define

$$
r^{\mathfrak{l}}(\lambda)=\sum_{\substack{\alpha>0 \\ e_{\alpha} \in \mathfrak{l}}} \frac{e_{\alpha} \otimes e_{-\alpha}-e_{-\alpha} \otimes e_{\alpha}}{(\lambda, \alpha)} .
$$

It is clear that this is the image of the basic rational dynamical r-matrix of $\mathfrak{l}$ under the embedding $\mathfrak{l} \subset \mathfrak{g}$.

Theorem 4.1 ([EV1] $)$. Any classical dynamical r-matrix $r: \mathfrak{h}^{*} \rightarrow(\mathfrak{g} \otimes \mathfrak{g})^{\mathfrak{h}}$ with zero coupling constant is gauge-equivalent to $r^{\mathfrak{l}}(\lambda)$ for some $\mathfrak{l}$.

4.3. Classification of dynamical r-matrices with coupling constant $\varepsilon \in \mathbb{C}^{*}$. Let $X \subset \Pi$, and denote by $\langle X\rangle \subset \Delta$ the set of all roots which are linear combinations of elements in $X \cup-X$. For any $\alpha \in \Delta$ introduce a meromorphic function $\varphi_{\alpha}: \mathfrak{h}^{*} \rightarrow \mathbb{C}$ by the following rule. Set $\varphi_{\alpha}(\lambda)=\frac{\varepsilon}{2}$ if $\alpha \in \Delta^{+} \backslash\langle X\rangle, \varphi_{\alpha}(\lambda)=-\frac{\varepsilon}{2}$ if $\alpha \in \Delta^{-} \backslash\langle X\rangle$ and

$$
\varphi_{\alpha}(\lambda)=\frac{\varepsilon}{2} \operatorname{cotanh}\left(\frac{\varepsilon}{2}(\lambda, \alpha)\right)
$$

if $\alpha \in\langle X\rangle$.

Theorem 4.2 ([EV1] $)$. Let $X \subset \Pi$. Set

$$
r_{X}(\lambda)=\frac{\varepsilon}{2} \Omega+\sum_{\alpha \in \Delta} \varphi_{\alpha}(\lambda) e_{\alpha} \otimes e_{-\alpha}
$$

Then $r_{X}^{\varepsilon}(\lambda)$ is a classical dynamical $r$-matrix with coupling constant $\varepsilon$. Moreover, any classical dynamical $r$-matrix with coupling constant $\varepsilon$ is gauge-equivalent to $r_{X}^{\varepsilon}(\lambda)$ for a suitable $X \subset \Pi$.

Remarks. 1. The basic trigonometric dynamical r-matrix $r^{\varepsilon}(\lambda)$ is obtained when we take $X=\Pi$. Moreover, the r-matrix $r_{X}^{\varepsilon}(\lambda)$ is equal to a limit of $r^{\varepsilon}(\lambda-\nu)$ when $\nu$ tends to infinity in $\mathfrak{h}^{*}$ in an appropriate direction. In other words, every classical dynamical r-matrix with nonzero coupling constant $\varepsilon$ is a limiting case of the basic trigonometric r-matrix.

2. Let $W$ be the Weyl group of $\mathfrak{g}$, and let $w \in W$. Let $\lambda \in \mathfrak{h}^{*}$ tend to infinity in a generic way in the Weyl chamber associated to $w$. Then

$$
\lim r^{1}(\lambda)=\frac{1}{2} \sum_{i} x_{i} \otimes x_{i}+\sum_{\alpha \in w\left(\Delta^{+}\right)} e_{\alpha} \otimes e_{-\alpha}
$$

which is the standard classical r-matrix corresponding to the polarization of $\mathfrak{g}$ associated to $w$. Hence the basic trigonometric dynamical r-matrix $r^{1}(\lambda)$ interpolates all $\mathfrak{h}$-invariant classical (non-dynamical) r-matrices $r$ satisfying $r+$ $r^{21}=\Omega$, (up to the addition of a skew 2 -form in $\Lambda^{2} \mathfrak{h}$ ). 
A classification of all classical dynamical r-matrices $r: \mathfrak{l}^{*} \rightarrow(\mathfrak{g} \otimes \mathfrak{g})^{\mathfrak{l}}$ where $\mathfrak{g}$ is a simple Lie algebra and $\mathfrak{l} \subset \mathfrak{h}$ is given in $|\mathbb{S}|$. This classification generalizes both the above classification (when $\mathfrak{l}=\mathfrak{h}$ ) and the Belavin-Drinfeld classification of classical r-matrices (when $\mathfrak{l}=0$ ) (see Appendix A).

\section{Classical dynamical r-matrices and Poisson- Lie groupoids}

In this section we give a geometric interpretation of the CDYBE. Let us first briefly recall the relationship between the classical Yang-Baxter equation and the theory of Poisson-Lie groups, developed by Drinfeld.

5.1. Poisson-Lie groups. Let $G$ be a (complex or real) Lie group, let $\mathfrak{g}$ be its Lie algebra and let $\mathcal{O}(G)$ be the algebra of regular functions on $G$. Let $\{\}:, \mathcal{O}(G) \times \mathcal{O}(G) \rightarrow \mathcal{O}(G)$ be a Poisson structure on $G$. Let $\Pi$ be the Poisson bivector field, defined by the relation $\{f, g\}=d f \otimes d g(\Pi)$. Recall that $(G,\{\}$, is called a Poisson-Lie group if the multiplication map $m: G \times G \rightarrow G$ is a Poisson map.

Let $\rho \in \Lambda^{2} \mathfrak{g}$ and consider the following bivector field:

$$
\Pi_{\rho}=R_{\rho}-L_{\rho}
$$

where $R_{\rho}$ (resp. $L_{\rho}$ ) is the left-invariant (resp. right-invariant) bivector field satisfying $\left(R_{\rho}\right)_{\mid e}=\rho\left(\operatorname{resp} .\left(L_{\rho}\right)_{\mid e}=\rho\right)$; in other words, $R_{\rho}, L_{\rho}$ stand for the translates of $\rho$ by right and left shifts respectively.

Proposition 5.1 (Drinfeld). The bivector $\Pi_{\rho}$ defines a Poisson-Lie group structure on $G$ if and only if

$$
\left[\rho_{12}, \rho_{13}\right]+\left[\rho_{13}, \rho_{23}\right]+\left[\rho_{12}, \rho_{23}\right] \in\left(\Lambda^{3} \mathfrak{g}\right)^{\mathfrak{g}} .
$$

When this is the case, $G$ is called a coboundary Poisson-Lie group. Two cases are of special interest:

1. The exists $T \in\left(S^{2} \mathfrak{g}\right)^{\mathfrak{g}}$ such that $\left[\rho_{12}, \rho_{13}\right]+\left[\rho_{13}, \rho_{23}\right]+\left[\rho_{12}, \rho_{23}\right]=\frac{1}{4}\left[T_{12}, T_{23}\right]$. This implies that $r=\rho+\frac{1}{2} T$ satisfies the classical Yang-Baxter equation, and $\Pi_{\rho}=R_{r}-L_{r}$. In this case $G$ is called a quasitriangular Poisson-Lie group.

2. We have $\left[\rho_{12}, \rho_{13}\right]+\left[\rho_{13}, \rho_{23}\right]+\left[\rho_{12}, \rho_{23}\right]=0$. In this case, $G$ is called a triangular Poisson-Lie group.

5.2. Poisson-Lie groupoids. It turns out that, in order to generalize this correspondence to the dynamical case, groups must be replaced by groupoids. Recall that a groupoid is a (small) category where all morphisms are isomorphisms. It is equivalent to the following data: two sets $X$ and $P$ (the set of morphisms, or the groupoid itself, and the set of objects, or the base, respectively), two surjective maps $s, t: X \rightarrow P$ (the source and target maps), an injective map $E: P \rightarrow X$ (the identity morphisms), a map $m:\{(a, b) \in X \times X \mid t(a)=$ $s(b)\} \rightarrow X(m(a, b)=b \circ a$, the composition of morphisms), and an involution 
$i: X \rightarrow X$ such that $s(i(x))=t(x), t(i(x))=s(x), m(i(x), x)=\operatorname{Id}_{s(x)}$ and $m(x, i(x))=\operatorname{Id}_{t(x)}$ for all $x \in X$, satisfying some obvious axioms. One can visualize elements of $X$ as arrows $s(a) \stackrel{a}{\rightarrow} t(a)$.

Note that when $|P|=1$, the notion of a groupoid coincides with the notion of a group.

A Lie groupoid is a groupoid with a smooth structure (in particular, the sets of objects and the sets of morphisms are smooth manifolds and the structure maps are smooth, see [M]).

Now we would like to generalize the notion of a Poisson-Lie group to groupoids. The usual definition does not generalize directly since if $X$ is a Lie groupoid and a Poisson manifold then the set of points $(a, b) \in X^{2}$ for which the multiplication is defined is not necessarily a Poisson submanifold, so we cannot require that the multiplication map be Poisson. But this difficulty can be bypassed using the following observation:

Proposition 5.2. Let $X, Y$ be two Poisson manifolds and let $f: X \rightarrow Y$ be a smooth map. Consider the graph $\Gamma_{f}=\{(x, f(x))\} \subset X \times \bar{Y}$, where $\bar{Y}$ is the manifold $Y$, with the opposite Poisson structure $\{,\}_{\bar{Y}}=-\{,\}_{Y}$. Then $f$ is a Poisson map if and only if $\Gamma_{f}$ is a coisotropic submanifold of $X \times \bar{Y}$, i.e if and only if for any $z \in \Gamma_{f},\left(T_{z} \Gamma_{f}\right)^{\perp} \subset T_{z}^{*}(X \times \bar{Y})$ is an isotropic subspace with respect to the Poisson form $\Pi$ on $T_{z}(X \times \bar{Y})^{*}$.

This gives rise to the following notion of a Poisson-Lie groupoid, first introduced by Weinstein $[$ ].

Definition. A Lie groupoid $X$ with a Poisson structure is called a Poisson-Lie groupoid if $\Gamma_{m} \subset X \times X \times \bar{X}$ is a coisotropic submanifold.

We now restrict ourselves to a particular class of Lie groupoids. Let $G$ be a Lie group, let $\mathfrak{g}$ be its Lie algebra, $\mathfrak{h} \subset \mathfrak{g}$ a subalgebra and $H$ a Lie subgroup of $G$ with Lie algebra $\mathfrak{h}$. Let $U \subset \mathfrak{h}^{*}$ be an open set. Consider the following groupoid: $X=U \times G \times U, P=U$ with $s\left(u_{1}, g, u_{2}\right)=u_{1}, t\left(u_{1}, g, u_{2}\right)=u_{2}$. The composition $m\left(\left(u_{1}, g, u_{2}\right),\left(u_{3}, g^{\prime}, u_{4}\right)\right)$ is defined only when $u_{2}=u_{3}$ and $m\left(\left(u_{1}, g, v\right),\left(v, g^{\prime}, u_{4}\right)\right)=\left(u_{1}, g g^{\prime}, u_{4}\right)$. If $a$ is a function on $U$ we set $a_{1}=$ $s^{*}(a) \in \mathcal{O}(X)$ and $a_{2}=t^{*}(a) \in \mathcal{O}(X)$. Let $\rho: U \rightarrow \Lambda^{2} \mathfrak{g}$ be a regular function.

The group $H^{2}$ acts on $X$ by

$$
\left(h_{1}, h_{2}\right)\left(u_{1}, g, u_{2}\right)=\left(\operatorname{Ad}^{*}\left(h_{1}\right) u_{1}, h_{1} g h_{2}^{-1}, \operatorname{Ad}^{*}\left(h_{2}\right) u_{2}\right) .
$$

We want to define a Poisson structure on $X$ for which $(-s, t)$ is a moment map for this action. This forces the following relations

$$
\begin{gathered}
\left\{a_{1}, b_{1}\right\}=-[a, b]_{1}, \quad\left\{a_{2}, b_{2}\right\}=[a, b]_{2}, \quad\left\{a_{1}, b_{2}\right\}=0, \\
\left\{a_{1}, f\right\}=R_{a} f, \quad\left\{a_{2}, f\right\}=L_{a} f .
\end{gathered}
$$

We try to complete the definition of the Poisson structure on $X$ by adding the relation

$$
\{f, g\}=(d f \otimes d g)\left(R_{\rho\left(u_{1}\right)}-L_{\rho\left(u_{2}\right)}\right),
$$


where $f, g$ are any functions on $X$ pulled back from $G$ and $a, b$ are linear functions on $U$.

Proposition $5.3(\sqrt{E V 1})$. Formulae $\sqrt{5.1}$ ) and $(5.3)$ define a Poisson-Lie groupoid structure on $X$ if and only if

1.

$$
\sum_{i}\left(x_{i}^{(1)} \frac{\partial \rho^{23}}{\partial x^{i}}-x_{i}^{(2)} \frac{\partial \rho^{13}}{\partial x^{i}}+x_{i}^{(3)} \frac{\partial \rho^{12}}{\partial x^{i}}\right)+\left[\rho^{12}, \rho^{13}\right]+\left[\rho^{12}, \rho^{23}\right]+\left[\rho^{13}, \rho^{23}\right]
$$

is a constant $\mathfrak{g}$-invariant element of $\Lambda^{3} \mathfrak{g}$, and

2. $\rho$ is $\mathfrak{h}$-invariant.

When this is the case, $X$ is called a coboundary dynamical Poisson-Lie groupoid, which will be denoted by $X_{r}$. Two cases are of special interest:

1. The exists $T \in\left(S^{2} \mathfrak{g}\right)^{\mathfrak{g}}$ such that

$$
\begin{aligned}
\frac{1}{4}\left[T_{12}, T_{23}\right]=\sum_{i} & \left(x_{i}^{(1)} \frac{\partial r^{23}(\lambda)}{\partial x^{i}}-x_{i}^{(2)} \frac{\partial r^{13}(\lambda)}{\partial x^{i}}+x_{i}^{(3)} \frac{\partial r^{12}(\lambda)}{\partial x^{i}}\right) \\
& +\left[\rho_{12}, \rho_{13}\right]+\left[\rho_{13}, \rho_{23}\right]+\left[\rho_{12}, \rho_{23}\right] .
\end{aligned}
$$

This implies that $r=\rho+\frac{1}{2} T$ satisfies the classical dynamical Yang-Baxter equation. In this case $X_{r}$ is called a quasitriangular dynamical Poisson-Lie groupoid.

2. We have

$$
\begin{aligned}
\sum_{i}\left(x_{i}^{(1)} \frac{\partial r^{23}(\lambda)}{\partial x^{i}}\right. & \left.-x_{i}^{(2)} \frac{\partial r^{13}(\lambda)}{\partial x^{i}}+x_{i}^{(3)} \frac{\partial r^{12}(\lambda)}{\partial x^{i}}\right) \\
& +\left[\rho_{12}, \rho_{13}\right]+\left[\rho_{13}, \rho_{23}\right]+\left[\rho_{12}, \rho_{23}\right]=0 .
\end{aligned}
$$

In this case, $X_{r}$ is called a triangular dynamical Poisson-Lie groupoid.

Thus, the basic rational solution defined above gives rise to a triangular dynamical Poisson-Lie groupoid, and the basic trigonometric solution gives rise to a quasitriangular one.

\section{Classification of quantum dynamical R-matrices}

In this section we give the classification of all quantum dynamical $R$-matrices $R: \mathfrak{h}^{*} \rightarrow \operatorname{End}_{\mathfrak{h}}(V \otimes V)$, where $\mathfrak{h}$ is the Cartan subalgebra of $\mathfrak{g l}(n, \mathbb{C})$ consisting of diagonal matrices, and $V=\mathbb{C}^{n}$ is the vector representation, which satisfy an additional Hecke condition, a quantum analogue of the unitarity condition.

6.1. Hecke condition. Let $\mathfrak{h}$ be the abelian Lie algebra of diagonal $\mathrm{N}$ by $\mathrm{N}$ matrices, and let $V$ be the standard $\mathrm{N}$-dimensional $\mathfrak{h}$-module. Let $h_{1}, \ldots h_{n}$ be the standard basis of $\mathfrak{h}, \lambda_{1}, \ldots \lambda_{n}$ be the corresponding coordinate functions on $\mathfrak{h}^{*}$, and $V_{i}, i=1, \ldots n$ be the (one-dimensional) weight subspaces of $V$ of weight $\omega_{i}$ where $\left\langle\omega_{i}, h_{j}\right\rangle=\delta_{i j}$.

Consider the h-module $V \otimes V$. Its weight subspaces are $V_{a} \otimes V_{b} \oplus V_{b} \otimes V_{a}$ and $V_{a} \otimes V_{a}$. 
Definition. An operator $R: \mathfrak{h}^{*} \rightarrow \operatorname{End}_{\mathfrak{h}}(V \otimes V)$ satisfies the Hecke condition with parameter $q \in \mathbb{C}^{*}$ if the eigenvalues of $P R$ (where $P$ is the permutation matrix) are 1 on $V_{a} \otimes V_{a}$ and $1,-q$ on $V_{a} \otimes V_{b} \oplus V_{b} \otimes V_{a}$.

This condition can be thought of as a quantum analogue of the unitarity condition for classical r-matrices, since it is easy to show that the quasiclassical limit of an operator satisfying the Hecke condition satisfies the unitarity condition. In particular if $R$ satisfies the Hecke condition with $q=1$ then $R R^{21}=1$, which can be thought of as a quantization of the relation $r+r^{21}=0$.

The terminology comes from the following remark: if $R$ is a $\lambda$-independent solution of the quantum dynamical Yang-Baxter equation satisfying the Hecke condition with parameter $q$ then $\check{R}$ defines a representation of the Hecke algebra $H_{p}$ of type $A_{p-1}$ on the space $V^{\otimes p}$ for any $p>1$. Similar representations can be defined for dynamical R-matrices (see [EV2] and Section 7).

6.2. Gauge transformations. Let $R(\lambda)$ be a quantum dynamical R-matrix satisfying Hecke condition with parameter $q$. The weight-zero and Hecke conditions imply that

$$
R(\lambda)=\sum_{a} E_{a a} \otimes E_{a a}+\sum_{a \neq b} \alpha_{a b}(\lambda) E_{a a} \otimes E_{b b}+\sum_{a \neq b} \beta_{a b}(\lambda) E_{a b} \otimes E_{b a}
$$

where $E_{i j}$ is the elementary matrix, and $\alpha_{a b}, \beta_{a b}$ are meromorphic functions $\mathfrak{h}^{*} \rightarrow \mathbb{C}$. So it is enough to look for solutions of this form.

As in the classical case, we will give the classification of solutions up to some group of transformations.

Definition. A multiplicative 2-form on $V$ is a collection meromorphic functions $\left\{\varphi_{a b}: \mathfrak{h}^{*} \rightarrow \mathbb{C}\right\}_{a, b=1}^{n}$ satisfying $\varphi_{a b} \varphi_{b a}=1$ for all $a, b$. A multiplicative 2 -form $\left\{\varphi_{a b}(\lambda)\right\}$ is closed if for all $a, b, c$,

$$
\frac{\varphi_{a b}(\lambda)}{\varphi_{a b}\left(\lambda-\omega_{c}\right)} \frac{\varphi_{b c}(\lambda)}{\varphi_{b c}\left(\lambda-\omega_{a}\right)} \frac{\varphi_{c a}(\lambda)}{\varphi_{c a}\left(\lambda-\omega_{b}\right)}=1 .
$$

Consider the following operations on meromorphic weight-zero maps $R: \mathfrak{h}^{*} \rightarrow$ $\operatorname{End}_{\mathfrak{h}}(V \otimes V)$ of the form (6.1):

1.

$$
R(\lambda) \mapsto \sum_{a} E_{a a} \otimes E_{a a}+\sum_{a \neq b} \varphi_{a b}(\lambda) \alpha_{a b}(\lambda) E_{a a} \otimes E_{b b}+\sum_{a \neq b} \beta_{a b}(\lambda) E_{a b} \otimes E_{b a},
$$

where $\left\{\varphi_{a b}(\lambda)\right\}$ is a closed multiplicative 2-form on $V$,

2. $R(\lambda) \mapsto R(\lambda-\nu)$ where $\nu$ is a pseudoconstant, i.e. a meromorphic function $\mathfrak{h}^{*} \rightarrow \mathfrak{h}^{*}$ such that $\nu\left(\lambda+\omega_{i}\right)=\nu(\lambda)$ for all $i$ (for example, a constant),

3. $R(\lambda) \mapsto(\sigma \otimes \sigma) R\left(\sigma^{-1} \lambda\right)\left(\sigma^{-1} \otimes \sigma^{-1}\right)$ where $\sigma \in \mathfrak{S}_{n}$ acts on $V$ and $\mathfrak{h}^{*}$ by permutation of coordinates. 
Remark. Here we allow to perform transformation 2 only if the answer is meromorphic.

Lemma 6.1. Tranformations $1-3$ preserve the set of quantum dynamical $R$ matrices.

Two R-matrices which can be obtained one from the other by a sequence of such transformations are said to be gauge-equivalent.

6.3. Classification for $q=1$. Let $X$ be a subset of $\{1, \ldots n\}$ and write $X=X_{1} \cup \ldots \cup X_{k}$ where $X_{i}=\left\{a_{i} \ldots b_{i}\right\}$ are disjoint intervals. Set

$$
R_{X}(\lambda)=\sum_{a, b=1}^{n} E_{a a} \otimes E_{b b}+\sum_{l=1}^{k} \sum_{\substack{a, b \in X_{l} \\ a \neq b}} \frac{1}{\lambda_{a}-\lambda_{b}}\left(E_{a a} \otimes E_{b b}+E_{b a} \otimes E_{a b}\right) .
$$

Theorem 6.1 ([EV2]). Let $X \subset\{1, \ldots n\}$. Then $R_{X}(\lambda)$ is a quantum $d y$ namical R-matrix satisfying the Hecke condition with $q=1$. Moreover, any dynamical $R$-matrix $R: \mathfrak{h}^{*} \rightarrow \operatorname{End}_{\mathfrak{h}}(V \otimes V)$ is gauge-equivalent to $R_{X}(\lambda)$ for a unique subset $X \subset\{1, \ldots n\}$.

Remark. The function $R_{X}(\lambda / \gamma)$ is, up to a gauge transformation, a quantization in the sense of Section 3 of the rational classical dynamical r-matrix (4.1) corresponding to the reductive subalgebra of $\mathfrak{g l}(n)$ spanned by root subspaces $\mathfrak{g}_{\alpha}, \mathfrak{g}_{-\alpha}$ for $\alpha \in X$.

The most interesting solution $R_{X}$ corresponds to the case when $X=\{1, \ldots, n\}$. We will call it the basic rational solution of the QDYBE.

6.4. Classification for $q \neq 1$. Let $\varepsilon \notin 2 i \pi \mathbb{Z}$ and set $q=e^{\varepsilon}$. Let $X$ be a subset of $\{1, \ldots n\}$ and again write $X=X_{1} \cup \ldots \cup X_{k}$ where $X_{i}=\left\{a_{i} \ldots b_{i}\right\}$ are disjoint intervals. Set

$$
R_{X}^{\varepsilon}(\lambda)=\sum_{a} E_{a a} \otimes E_{a a}+\sum_{a \neq b} \alpha_{a b}(\lambda) E_{a a} \otimes E_{b b}+\sum_{a \neq b} \beta_{a b}(\lambda) E_{a b} \otimes E_{b a},
$$

where $\alpha_{a b}(\lambda)=q+\beta_{a b}(\lambda)$ and where $\beta_{a b}(\lambda)$ is defined as follows: $\beta_{a b}=\frac{q-1}{q^{\lambda_{a}-\lambda_{b}-1}}$ if $a, b \in X_{l}$ for some $1 \leq l \leq k, \beta_{a b}(\lambda)=1-q$ otherwise if $a>b$ and $\beta_{a b}(\lambda)=0$ otherwise if $a<b$.

Theorem 6.2 ([EV2]). Let $X \subset\{1, \ldots n\}$. Then $R_{X}^{\varepsilon}(\lambda)$ is a quantum $d y$ namical R-matrix satisfying the Hecke condition with $q=e^{\varepsilon}$. Moreover, any dynamical R-matrix $R: \mathfrak{h}^{*} \rightarrow \operatorname{End}_{\mathfrak{h}}(V \otimes V)$ is gauge-equivalent to $R_{X}(\lambda)$ for a unique subset $X \subset\{1, \ldots n\}$.

Remark. It can be checked that the $R_{X}^{\varepsilon}(\lambda)$ yield (again up to gauge transformations) quantizations of the trigonometric classical dynamical r-matrices with coupling constant $\varepsilon$ appearing in Theorem 4.2.

The most interesting solution $R_{X}^{\varepsilon}$ corresponds to the case when $X=\{1, \ldots, n\}$. We will call it the basic trigonometric solution of the QDYBE. 
6.5. The fusion and exchange matrices for the vector representation of classical and quantum $g l_{n}$. The above classification can be applied to compute the fusion and exchange matrices for the vector representation. Namely, we have:

Theorem 6.3 $([\overline{E V 3}])$. 1. Let $\mathfrak{g}=\mathfrak{g l}_{n}$ and let $V=\mathbb{C}^{n}$ be the vector representation. Then

$$
\begin{aligned}
J_{V V}(\lambda)= & +\sum_{a<b} \frac{1}{\lambda_{b}-\lambda_{a}+a-b} E_{b a} \otimes E_{a b} \\
R_{V V}(\lambda)= & \sum_{a=1}^{n} E_{a a} \otimes E_{a a}+\sum_{a \neq b} \frac{1}{\lambda_{a}-\lambda_{b}+b-a} E_{b a} \otimes E_{a b}+\sum_{a<b} E_{a a} \otimes E_{b b} \\
& \quad-\sum_{a>b} \frac{\left(\lambda_{b}-\lambda_{a}+a-b-1\right)\left(\lambda_{b}-\lambda_{a}+a-b+1\right)}{\left(\lambda_{b}-\lambda_{a}+a-b\right)^{2}} E_{a a} \otimes E_{b b} .
\end{aligned}
$$

2. Let $V=\mathbb{C}^{n}$ be the representation of $U_{q}\left(\mathfrak{g} l_{N}\right)$ which is the q-analog of the vector representation. Then

$$
\begin{aligned}
J_{V V}(\lambda)=1 & +\sum_{a<b} \frac{q^{-1}-q}{q^{2\left(\lambda_{a}-\lambda_{b}+b-a\right)}-1} E_{b a} \otimes E_{a b} \\
R_{V V}(\lambda)=q & \sum_{a=1}^{n} E_{a a} \otimes E_{a a}+\sum_{a \neq b} \frac{q^{-1}-q}{q^{2\left(\lambda_{a}-\lambda_{b}+b-a\right)}-1} E_{b a} \otimes E_{a b}+\sum_{a<b} E_{a a} \otimes E_{b b} \\
& +\sum_{a>b} \frac{\left(q^{2\left(\lambda_{b}-\lambda_{a}+a-b\right)}-q^{-2}\right)\left(q^{2\left(\lambda_{b}-\lambda_{a}+a-b\right)}-q^{2}\right)}{\left(q^{2\left(\lambda_{b}-\lambda_{a}+a-b\right)}-1\right)^{2}} E_{a a} \otimes E_{b b} .
\end{aligned}
$$

Proof. The proof relies on explicit computations and on the classification of quantum dynamical R-matrices (Theorems 6.1 and 6.2). More precisely, it is possible to compute explicitly the coefficients of $J$ correpsonding to simple roots, and all the other coefficients are then uniquely determined by Theorems 6.1 and 6.2 .

Remark. The matrix coefficients of $J_{V V}(\lambda)$ for nonsimple roots are not as easily computed directly as those for simple roots. The above approach allows one to avoid this calculation.

\section{Quantum dynamical R-matrices and quantum groupoids}

In this section we will give a "noncommutative geometric" interpretation of the QDYBE which is analogous to the geometric interpretation of the CDYBE given above. More precisely, to solutions of the QDYBE we will associate, following [E], EV2], a kind of quantum group, more precisely a Hopf algebroid (or quantum groupoid).

The general notion of a Hopf algebroid was introduced in $[\mathrm{Lu}]$. However, here it will be sufficient to use a less general notion, that of an $H$-Hopf algebroid, which was introduced in [EV2]. Our exposition will follow EV2, EV3]. 
7.1. $H$-bialgebroids. Let $H$ be a commutative and cocommutative finitely generated Hopf algebra over $\mathbb{C}, T=\operatorname{Spec} H$ the corresponding commutative affine algebraic group. Assume that $T$ is connected. Let $M_{T}$ denote the field of meromorphic functions on $T$. Let us introduce the following definitions.

Definition. $A n H$-algebra is an associative algebra $A$ over $\mathbb{C}$ with 1 , endowed with an $T$-bigrading $A=\oplus_{\alpha, \beta \in T} A_{\alpha \beta}$ (called the weight decomposition), and two algebra embeddings $\mu_{l}, \mu_{r}: M_{T} \rightarrow A_{00}$ (the left and the right moment maps), such that for any $a \in A_{\alpha \beta}$ and $f \in M_{T}$, we have

$$
\mu_{l}(f(\lambda)) a=a \mu_{l}(f(\lambda+\alpha)), \quad \mu_{r}(f(\lambda)) a=a \mu_{r}(f(\lambda+\beta)) .
$$

$A$ morphism $\varphi: A \rightarrow B$ of two $H$-algebras is an algebra homomorphism, preserving the moment maps.

Example 1. Let $D_{T}$ be the algebra of difference operators $M_{T} \rightarrow M_{T}$, i.e. the operators of the form $\sum_{i=1}^{n} f_{i}(\lambda) \mathcal{T}_{\beta_{i}}$, where $f_{i} \in M_{T}$, and for $\beta \in T$ we denote by $\mathcal{T}_{\beta}$ the field automorphism of $M_{T}$ given by $\left(\mathcal{T}_{\beta} f\right)(\lambda)=f(\lambda+\beta)$.

The algebra $D_{T}$ is an example of an $H$-algebra if we define the weight decomposition by $D_{T}=\oplus\left(D_{T}\right)_{\alpha \beta}$, where $\left(D_{T}\right)_{\alpha \beta}=0$ if $\alpha \neq \beta$, and $\left(D_{T}\right)_{\alpha \alpha}=$ $\left\{f(\lambda) \mathcal{T}_{\alpha}^{-1}: f \in M_{T}\right\}$, and the moment maps $\mu_{l}=\mu_{r}: M_{T} \rightarrow\left(D_{T}\right)_{00}$ to be the tautological isomorphism.

Example 2. This is a generalization of Example 1. Let $W$ be a diagonalizable $H$-module, $W=\oplus_{\lambda \in T} W[\lambda], W[\lambda]=\{w \in W \mid a w=\lambda(a) w$, for all $a \in H\}$, and let $D_{T, W}^{\alpha} \subset \operatorname{Hom}_{\mathbb{C}}\left(W, W \otimes D_{T}\right)$ be the space of all difference operators on $T$ with coefficients in End $\mathbb{C}_{\mathbb{C}}(W)$, which have weight $\alpha \in T$ with respect to the action of $H$ in $W$.

Consider the algebra $D_{T, W}=\oplus_{\alpha} D_{T, W}^{\alpha}$. This algebra has a weight decomposition $D_{T, W}=\oplus_{\alpha, \beta}\left(D_{T, W}\right)_{\alpha \beta}$ defined as follows: if $g \in \operatorname{Hom}_{\mathbb{C}}\left(W, W \otimes M_{T}\right)$ is an operator of weight $\beta-\alpha$, then $g \mathcal{T}_{\beta}^{-1} \in\left(D_{T, W}\right)_{\alpha \beta}$.

Define the moment maps $\mu_{l}, \mu_{r}: M_{T} \rightarrow\left(D_{T, W}\right)_{00}$ by the formulas $\mu_{r}(f(\lambda))=$ $f(\lambda), \mu_{l}(f(\lambda))=f(\lambda-h)$ where $f(\lambda-h) w=f(\lambda-\mu) w$ if $w \in W[\mu], \mu \in T$. The algebra $D_{T, W}$ equipped with this weight decomposition and these moment maps is an $H$-algebra.

Now let us define the tensor product of $H$-algebras. Let $A, B$ be two $H$ algebras and $\mu_{l}^{A}, \mu_{r}^{A}, \mu_{l}^{B}, \mu_{r}^{B}$ their moment maps. Define their matrix tensor product, $A \widetilde{\otimes} B$, which is also an $H$-algebra. Let

$$
(A \widetilde{\otimes} B)_{\alpha \delta}:=\oplus_{\beta} A_{\alpha \beta} \otimes_{M_{T}} B_{\beta \delta},
$$

where $\otimes_{M_{T}}$ means the usual tensor product modulo the relation $\mu_{r}^{A}(f) a \otimes b=$ $a \otimes \mu_{l}^{B}(f) b$, for any $a \in A, b \in B, f \in M_{T}$. Introduce a multiplication in $A \widetilde{\otimes} B$ by the rule $(a \otimes b)\left(a^{\prime} \otimes b^{\prime}\right)=a a^{\prime} \otimes b b^{\prime}$. It is easy to check that the multiplication is well defined. Define the moment maps for $A \widetilde{\otimes} B$ by $\mu_{l}^{A \tilde{\otimes} B}(f)=\mu_{l}^{A}(f) \otimes 1$, $\mu_{r}^{A \tilde{\otimes} B}(f)=1 \otimes \mu_{r}^{B}(f)$.

For any $H$-algebra $A$, the algebras $A \widetilde{\otimes} D_{T}$ and $D_{T} \widetilde{\otimes} A$ are canonically isomorphic to $A$. In particular, $D_{T}$ is canonically isomorphic to $D_{T} \widetilde{\otimes} D_{T}$. Thus 
the category of $H$-algebras equipped with the product $\widetilde{\otimes}$ is a monoidal category, where the unit object is $D_{T}$.

Now let us define the notions of a coproduct and a counit on an $H$-algebra.

Definition. $\quad A$ coproduct on an $H$-algebra $A$ is a homomorphism of $H$-algebras $\Delta: A \rightarrow A \widetilde{\otimes} A$.

$A$ counit on an $H$-algebra $A$ is a homomorphism of $H$-algebras $\epsilon: A \rightarrow D_{T}$.

Finally, we can define the notions of an $H$-bialgebroid and an $H$-Hopf algebroid.

Definition. An $H$-bialgebroid is an $H$-algebra $A$ equipped with a coassociative coproduct $\Delta$ (i.e. such that $\left(\Delta \otimes \operatorname{Id}_{A}\right) \circ \Delta=\left(\operatorname{Id}_{A} \otimes \Delta\right) \circ \Delta$, and a counit $\epsilon$ such that $\left(\epsilon \otimes \operatorname{Id}_{A}\right) \circ \Delta=\left(\operatorname{Id}_{A} \otimes \epsilon\right) \circ \Delta=\operatorname{Id}_{A}$.

Let $A$ be an $H$-algebra. A linear map $S: A \rightarrow A$ is called an antiautomorphism of $H$-algebras if it is an antiautomorphism of algebras and $\mu_{r} \circ S=$ $\mu_{l}, \mu_{l} \circ S=\mu_{r}$. From these conditions it follows that $S\left(A_{\alpha \beta}\right)=A_{-\beta,-\alpha}$.

Let $A$ be an $H$-bialgebroid, and let $\Delta, \epsilon$ be the coproduct and counit of $A$. For $a \in A$, let

$$
\Delta(a)=\sum_{i} a_{i}^{1} \otimes a_{i}^{2}
$$

Definition. An antipode on the $H$-bialgebroid $A$ is an antiautomorphism of $H$-algebras $S: A \rightarrow A$ such that for any $a \in A$ and any presentation (7.3) of $\Delta(a)$, one has

$$
\sum_{i} a_{i}^{1} S\left(a_{i}^{2}\right)=\mu_{l}(\epsilon(a) 1), \sum_{i} S\left(a_{i}^{1}\right) a_{i}^{2}=\mu_{r}(\epsilon(a) 1),
$$

where $\epsilon(a) 1 \in M_{T}$ is the result of the application of the difference operator $\epsilon(a)$ to the constant function 1.

An $H$-bialgebroid with an antipode is called an $H$-Hopf algebroid.

Remarks. $\quad 1$. If $H=\mathbb{C}$ then the notions of $H$-algebra, $H$-bialgebroid, $H$-Hopf algebroid are the familiar notions of an algebra, bialgebra, and Hopf algebra.

2. It is easy to see that $D_{T}$ is an $H$-bialgebroid where $\Delta: D_{T} \rightarrow D_{T} \tilde{\otimes} D_{T}$ is the canonical isomorphism and $\epsilon=\mathrm{Id}$. Furthermore, it is an $H$-Hopf algebroid with $S(D)=D^{*}$, where $D^{*}$ is the formal adjoint to the difference operator $D$ (i.e. $\left.\left(f(\lambda) \mathcal{T}_{\alpha}\right)^{*}=\mathcal{T}_{\alpha}^{-1} f(\lambda)\right)$. This $H$-Hopf algebroid is an analog of the 1-dimensional Hopf algebra in the category of Hopf algebras.

3 . One can define the notions of an $H$-algebra, $H$-bialgebroid, $H$-Hopf algebroid if the group $T$ is not connected (for example, a finite group), in essentially the same way as above. More precisely, since in this case the algebra $M_{T}$ of meromorphic functions on $T$ is not a field but a direct sum of finitely many copies of a field, one should introduce an additional axiom requiring that $A_{\alpha \beta}$ is a free module over $\mu_{l}\left(M_{T}\right)$ and $\mu_{r}\left(M_{T}\right)$. Similarly, one can make all the above definitions in the case when $M_{T}$ is replaced with another algebra of functions on $T$ (rational functions, regular functions on some open set, etc.) 
7.2. Dynamical representations of $H$-bialgebroids. One of the reasons $H$-bialgebroids are good analogs of bialgebras is that their representations, like representations of bialgebras, form a tensor category. However, these representations are not the usual representations but rather new objects which we call dynamical representations, and which we will now define.

Definition. A dynamical representation of an $H$-algebra $A$ is a diagonalizable $H$-module $W$ endowed with a homomorphism of $H$-algebras $\pi_{W}: A \rightarrow D_{T, W}$, where $D_{T, W}$ is defined in Example 2 .

Definition. A homomorphism of dynamical representations $\varphi: W_{1} \rightarrow W_{2}$ is an element of $\operatorname{Hom}_{\mathbb{C}}\left(W_{1}, W_{2} \otimes M_{T}\right)$ such that $\varphi \circ \pi_{W_{1}}(x)=\pi_{W_{2}}(x) \circ \varphi$ for all $x \in A$.

Example. If $A$ has a counit, then $A$ has the trivial representation: $W=\mathbb{C}$, $\pi=\epsilon$.

For diagonalizable $H$-modules $W, U$, let $f \in \operatorname{Hom}\left(W, W \otimes M_{T}\right)$ and $g \in \operatorname{Hom}\left(U, U \otimes M_{T}\right)$. Define $f \bar{\otimes} g \in \operatorname{Hom}\left(W \otimes U, W \otimes U \otimes M_{T}\right)$ as

$$
f \bar{\otimes} g(\lambda)=f^{(1)}\left(\lambda-h^{(2)}\right)(1 \otimes g(\lambda))
$$

where $f^{(1)}\left(\lambda-h^{(2)}\right)(1 \otimes g(\lambda)) w \otimes u=f(\lambda-\mu) w \otimes g(\lambda) u$ if $g(\lambda) u \in U[\mu]$.

Lemma 1 ([EV2]). There is a natural embedding of $H$-algebras $\theta_{W U}: D_{T, W} \otimes D_{T, U} \rightarrow D_{T, W \otimes U}$ (an isomorphism if $W, U$ are finite dimensional), given by the formula $f \mathcal{T}_{\beta} \otimes g \mathcal{T}_{\delta} \rightarrow(f \bar{\otimes} g) \mathcal{T}_{\delta}$.

Now let us define the tensor product of dynamical representations for $\mathrm{H}$ bialgebroids. If $A$ is an $H$-bialgebroid, and $W$ and $U$ are two dynamical representations of $A$, then we endow the $H$-module $W \otimes U$ with the structure of a dynamical representation via $\pi_{W \otimes U}(x)=\theta_{W U} \circ\left(\pi_{W} \otimes \pi_{U}\right) \circ \Delta(x)$. If $f: W_{1} \rightarrow W_{2}$ and $g: U_{1} \rightarrow U_{2}$ are homomorphisms of dynamical representations, then so is $f \bar{\otimes} g: W_{1} \otimes U_{1} \rightarrow W_{2} \otimes U_{2}$. Thus, dynamical representations of $A$ form a monoidal category $\operatorname{Rep}(A)$, whose identity object is the trivial representation.

Remark. If $A$ is an $H$-Hopf algebroid and $V$ is a dynamical representation, then one can define the left and right dual dynamical representations ${ }^{*} V$ and $V^{*}$. We will not discuss this notion here and refer the reader to [EV2].

7.3. The $H$-bialgebroid associated to a function $R: T \rightarrow \operatorname{End}(V \otimes V)$. Now, following [EV2], let us define an $H$-bialgebroid $\bar{A}_{R}$ associated to a meromorphic, zero weight function $R: T \rightarrow$ End $(V \otimes V)$, where $V$ is a finite dimensional diagonalizable $H$-module (we assume that $R(\lambda)$ is nondegenerate for generic $\lambda$ ). This is the dynamical analogue of the Faddeev-ReshetikhinTakhtajan-Sklyanin construction of a bialgebra from an element $R \in$ End $(V \otimes V)$, where $V$ is a vector space.

By definition, the algebra $\bar{A}_{R}$ is generated by two copies of $M_{T}$ (embedded as subalgebras) and matrix elements of the operator $L \in \operatorname{End}(V) \otimes \bar{A}_{R}$. We denote the elements of the first copy of $M_{T}$ by $f\left(\lambda^{1}\right)$ and of the second copy 
by $f\left(\lambda^{2}\right)$, where $f \in M_{T}$. We denote by $L_{\alpha \beta}$ the weight components of $L$ with respect to the natural $T$-bigrading on End $(V)$, so that $L=\left(L_{\alpha \beta}\right)$, where $L_{\alpha \beta} \in \operatorname{Hom}_{\mathbb{C}}(V[\beta], V[\alpha]) \otimes \bar{A}_{R}$.

Introduce the moment maps for $\bar{A}_{R}$ by $\mu_{l}(f)=f\left(\lambda^{1}\right), \mu_{r}(f)=f\left(\lambda^{2}\right)$, and define the weight decomposition by

$$
f\left(\lambda^{1}\right), f\left(\lambda^{2}\right) \in\left(\bar{A}_{R}\right)_{00}, \quad L_{\alpha \beta} \in \operatorname{Hom}_{\mathbb{C}}(V[\beta], V[\alpha]) \otimes\left(\bar{A}_{R}\right)_{\alpha \beta} .
$$

The defining relations for $\bar{A}_{R}$ are:

$$
f\left(\lambda^{1}\right) L_{\alpha \beta}=L_{\alpha \beta} f\left(\lambda^{1}+\alpha\right) ; f\left(\lambda^{2}\right) L_{\alpha \beta}=L_{\alpha \beta} f\left(\lambda^{2}+\beta\right) ;\left[f\left(\lambda^{1}\right), g\left(\lambda^{2}\right)\right]=0 ;
$$

and the dynamical Yang-Baxter relation

$$
R^{12}\left(\lambda^{1}\right) L^{13} L^{23}=: L^{23} L^{13} R^{12}\left(\lambda^{2}\right): .
$$

Here the :: sign means that the matrix elements of $L$ should be put on the right of the matrix elements of $R$. Thus, if $\left\{v_{a}\right\}$ is a homogeneous basis of $V$, and $L=\sum E_{a b} \otimes L_{a b}, R(\lambda)\left(v_{a} \otimes v_{b}\right)=\sum R_{c d}^{a b}(\lambda) v_{c} \otimes v_{d}$, then (7.5) has the form

$$
\sum R_{a c}^{x y}\left(\lambda^{1}\right) L_{x b} L_{y d}=\sum R_{x y}^{b d}\left(\lambda^{2}\right) L_{c y} L_{a x},
$$

where we sum over repeated indices.

Define the coproduct on $\bar{A}_{R}, \Delta: \bar{A}_{R} \rightarrow \bar{A}_{R} \tilde{\otimes} \bar{A}_{R}$, and the counit of $\bar{A}_{R}$ by

$$
\Delta(L)=L^{12} L^{13}, \epsilon\left(L_{\alpha \beta}\right)=\delta_{\alpha \beta} I d_{V[\alpha]} \otimes \mathcal{T}_{\alpha}^{-1},
$$

where $\operatorname{Id}_{V[\alpha]}: V[\alpha] \rightarrow V[\alpha]$ is the identity operator.

Proposition 7.1 (EV2]). $\left(\bar{A}_{R}, \Delta, \epsilon\right)$ is an $H$-bialgebroid.

Example. Suppose that $R$ is the basic trigonometric solution of the QDYBE (see Section 6 ). Then the defining relations for $\bar{A}_{R}$ look like

$$
\begin{aligned}
f\left(\lambda^{1}\right) L_{b c} & =L_{b c} f\left(\lambda^{1}+\omega_{b}\right), \\
f\left(\lambda^{2}\right) L_{b c} & =L_{b c} f\left(\lambda^{2}+\omega_{c}\right), \\
L_{a s} L_{a t} & =\frac{\alpha_{s t}\left(\lambda^{2}\right)}{1-\beta_{t s}\left(\lambda^{2}\right)} L_{a t} L_{a s}, s \neq t, \\
L_{b s} L_{a s} & =\frac{\alpha_{a b}\left(\lambda_{1}\right)}{1-\beta_{a b}\left(\lambda^{1}\right)} L_{a s} L_{b s}, a \neq b, \\
\alpha_{a b}\left(\lambda_{1}\right) L_{a s} L_{b t}-\alpha_{s t}\left(\lambda_{2}\right) L_{b t} L_{a s} & =\left(\beta_{t s}\left(\lambda_{2}\right)-\beta_{a b}\left(\lambda_{1}\right)\right) L_{b s} L_{a t}, a \neq b, s \neq t,
\end{aligned}
$$

where $\alpha_{a b}(\lambda)=\frac{q^{\lambda_{a}-\lambda_{b}+1}-1}{q^{\lambda_{a}-\lambda_{b}}-1}, \beta_{a b}(\lambda)=\frac{q-1}{q^{\lambda_{a}-\lambda_{b}-1}}$.

We note that we don't need any special properties of $R$ (like the dynamical Yang-Baxter equation or Hecke condition) to define the $H$-bialgebroid $\bar{A}_{R}$. However, if we take a "randomly chosen" function $R$, the $H$-bialgebroid $\bar{A}_{R}$ will most likely have rather bad properties; i.e. it will be rather small and will not have interesting dynamical representations. The simplest way to ensure the existence of at least one interesting dynamical representation is to require that $R$ satisfies the QDYBE. This is so because of the following proposition. 
If $\left(W, \pi_{W}\right)$ is a dynamical representation of an $H$-algebra $A$, we denote $\pi_{W}^{0}$ : $A \rightarrow \operatorname{Hom}\left(W, W \otimes M_{T}\right)$ the map defined by $\pi_{W}^{0}(x) w=\pi_{W}(x) w, w \in W$ (the difference operator $\pi_{W}(x)$ restricted to the constant functions). It is clear that $\pi_{W}$ is completely determined by $\pi_{W}^{0}$.

Proposition 7.2. If $R$ satisfies the $Q D Y B E$ then $\bar{A}_{R}$ has a dynamical representation realized in the space $V$, with $\pi_{V}^{0}(\lambda)=R(\lambda)$.

This representation is called the vector representation.

However, even if $R$ satisfies the QDYBE, the $H$-bialgebroid $\bar{A}_{R}$ may not be completely satisfactory. In particular, one may ask the following question: does $\bar{A}_{R}$ define a "good quantum matrix algebra"? More precisely, does the Hilbert series of $\bar{A}_{R}$ equal to that of the function ring on the matrix algebra, i.e. $(1-t)^{-\operatorname{dim}(V)^{2}}$ ? In general, the answer is no, even if the quantum dynamical Yang-Baxter equation is satisfied.

In fact, here is the place where the Hecke condition comes handy. Namely, we have the following proposition, which is a generalization of a well known proposition in the theory of quantum groups (due to Faddeev, Reshetikhin, Takhtajan).

Proposition 7.3 ([EV2]). Suppose that $R$ satisfies the QDYBE and the Hecke condition with $q$ not equal to a nontrivial root of 1 . Then the space $\bar{A}_{R}^{m}$ of polynomials of degree $m$ in generators $L_{\alpha \beta}$ in $\bar{A}_{R}$ is a free $M_{T} \otimes M_{T}$-module, and the ranks of these modules are given by

$$
\sum_{m \geq 0} r k\left(\bar{A}_{R}^{m}\right) t^{m}=(1-t)^{-n^{2}} .
$$

Remark. The proof of this proposition, like the proof of its nondynamical analog, is based on the fact that under the assumptions of the Proposition, $\bar{A}_{R}^{m}$ is a representation of the Hecke algebra. This justifies the name "Hecke condition".

7.4. The $H$-Hopf algebroid $A_{R}$. Suppose now that $R$ satisfies the QDYBE and the Hecke condition where $q$ is not a nontrivial root of 1 . In this case, it turns out that, analogously to the nondynamical case, a suitable localization $A_{R}$ of $\bar{A}_{R}$ is actually a Hopf algebroid. Namely, define $A_{R}$ by adjoining to $\bar{A}_{R}$ a new element $L^{-1}$, with the relation $L L^{-1}=L^{-1} L=1$. It is easy to see that the structure of an $H$-bialgebroid on $\bar{A}_{R}$ naturally extends to $A_{R}$, and it can be shown that $A_{R}$ admits a unique antipode $S$ such that $S(L)=L^{-1}$. This antipode equips $A_{R}$ with a structure of an $H$-Hopf algebroid. This $H$ Hopf algebroid is a quantization of the group $G L_{n}$ in the same sense as the $H$-bialgebroid $\bar{A}_{R}$ is a quantization of the matrix algebra $M_{a t}$.

7.5. Quasiclassical limit. In conclusion of the section, we would like to explain why the $H$-Hopf algebroid $A_{R}$ considered here (for the basic rational or trigonometric solution $R$ of the QDYBE) should be regarded as a quantization of the Poisson groupoid $X_{r}$ corresponding to the basic rational, respectively trigonometric, solution $r$ of the CDYBE (for the definition of $X_{r}$, see Section $5)$. 
To see this, consider the $H$-Hopf algebroid $A_{R}$ with $T=\mathfrak{h}^{*}$ for some finite dimensional abelian Lie algebra $\mathfrak{h}$, and $M_{T}$ replaced with the ring of regular functions on some open subset $U$ of $\mathfrak{h}^{*}$. Introduce a formal parameter $\gamma$ (like in Section 3), and make a change of variable $\lambda \rightarrow \lambda / \gamma$ in the defining relations for $A_{R}$. It is easy to see that the resulting algebra $A_{R}^{\gamma}$ over $\mathbb{C}[[\gamma]]$ is a deformation of a commutative algebra. The above result about the Hilbert series implies that this deformation is flat, so the quotient algebra $A_{R}^{0}:=A_{R}^{\gamma} /(\gamma)$ obtains a Poisson structure. Let $X$ be the spectrum of $A_{R}^{0}$; it is an algebraic Poisson manifold. It is not difficult to show that the the moment maps, coproduct, counit, and antipode of $A_{R}$ define maps $s, t, m, E, i$ (see Section 5) for $X$, which equips $X$ with the structure of a Poisson groupoid with base $U$. Moreover, it is easy to check that the Poisson groupoid $X$ is naturally isomorphic to $X_{r}$.

\section{The universal fusion matrix and the Arnaudon- Buffenoir-Ragoucy-Roche equation}

8.1. The ABRR equation. In ABRR, Arnaudon, Buffenoir, Ragoucy and Roche give a general method for constructing the universal fusion matrix $J(\lambda)$, which lives in some completion of $U_{q}(\mathfrak{g})^{\otimes 2}$, i.e the unique element satisfying $J_{V W}(\lambda)=J(\lambda)_{\mid V \otimes W}$ for all $V, W$. A similar approach is suggested in JKOS, based on the method of [F]

Let $U^{\prime}\left(\mathfrak{b}_{ \pm}\right)$be the kernel of the projection $U\left(\mathfrak{b}_{ \pm}\right) \rightarrow U(\mathfrak{h})$. We use the same notations with the index $q$ for the quantum analogs of these objects. We set $\theta(\lambda)=\lambda+\rho-\frac{1}{2} \sum_{i} x_{i}^{2} \in U \mathfrak{h}$ where as usual $\rho=\frac{1}{2} \sum_{\alpha \in \Delta^{+}} h_{\alpha}$ and $\left(x_{i}\right)$ is an orthonormal basis of $\mathfrak{h}$. Set $\mathcal{R}_{0}=\mathcal{R} q^{-\sum x_{i} \otimes x_{i}}$. It is known that $\mathcal{R}_{0} \in 1+U_{q}^{\prime}\left(\mathfrak{b}_{+}\right) \otimes U_{q}^{\prime}\left(\mathfrak{b}_{-}\right)$.

Theorem 8.1 ([ABRR]). The universal fusion matrix $J(\lambda)$ of $U_{q}(\mathfrak{g})$ is the unique solution of the form $1+U_{q}^{\prime}\left(\mathfrak{b}_{-}\right) \otimes U_{q}^{\prime}\left(\mathfrak{b}_{+}\right)$of the equation

$$
J(\lambda)\left(1 \otimes q^{2 \theta(\lambda)}\right)=\mathcal{R}_{0}^{21}\left(1 \otimes q^{2 \theta(\lambda)}\right) J(\lambda) .
$$

The universal fusion matrix $J(\lambda)$ of $U(\mathfrak{g})$ is the unique solution of the form $1+U^{\prime}\left(\mathfrak{b}_{-}\right) \otimes U^{\prime}\left(\mathfrak{b}_{+}\right)$of the equation

$$
[J(\lambda), 1 \otimes \theta(\lambda)]=\left(\sum_{\alpha \in \Delta^{+}} e_{-\alpha} \otimes e_{\alpha}\right) J(\lambda)
$$

We will call these equations the $A B R R$ equations for $U_{q}(\mathfrak{g})$ and for $\mathfrak{g}$, respectively.

Proof. Let us first show the statement about uniqueness. Let $T(\lambda) \in 1+$ $U_{q}^{\prime}\left(\mathfrak{b}_{-}\right) \otimes U_{q}^{\prime}\left(\mathfrak{b}_{+}\right)$be any solution of 8.1). Then

$$
\begin{aligned}
& \left(\mathcal{R}_{0}^{21}\right)^{-1} T(\lambda)=\operatorname{Ad}\left(1 \otimes q^{2 \theta(\lambda)}\right) T(\lambda) \\
& \Leftrightarrow\left(\left(\mathcal{R}_{0}^{21}\right)^{-1}-1\right) T(\lambda)=\left(\operatorname{Ad}\left(1 \otimes q^{2 \theta(\lambda)}\right)-1\right) T(\lambda) \\
& \quad \Leftrightarrow T(\lambda)=1+\left(\operatorname{Ad}\left(1 \otimes q^{2 \theta(\lambda)}\right)-1\right)^{-1}\left(\left(\mathcal{R}_{0}^{21}\right)^{-1}-1\right) T(\lambda)
\end{aligned}
$$

Now notice that $\left(\left(\mathcal{R}_{0}^{21}\right)^{-1}-1\right) \in U_{q}^{\prime}\left(\mathfrak{b}_{-}\right) \otimes U_{q}^{\prime}\left(\mathfrak{b}_{+}\right)$. This implies that $T(\lambda)$ can be recusively constructed as follows. Set $T_{0}(\lambda)=1$ and put

$$
T_{n+1}(\lambda)=1+\left(\operatorname{Ad}\left(1 \otimes q^{2 \theta(\lambda)}\right)-1\right)^{-1}\left(\left(\mathcal{R}_{0}^{21}\right)^{-1}-1\right) T_{n}(\lambda) .
$$


Then $\lim _{n \rightarrow \infty} T_{n}(\lambda)=T(\lambda)$ (the limit is in the sense of stabilization). In particular there exists a unique solution to (8.1) of the given form.

The proof in the rational case (i.e in the case of a simple Lie algebra $\mathfrak{g}$ ) is similar. In that case, the recursive construction is given by $T_{0}(\lambda)=1$ and

$$
T_{n+1}(\lambda)=1-\operatorname{ad}\left(1 \otimes \theta(\lambda)^{-1}\right)\left(\sum_{\alpha \in \Delta^{+}} e_{-\alpha} \otimes e_{\alpha}\right) T_{n}(\lambda) .
$$

We now give a proof that the fusion matrix $J(\lambda)$ actually satisfies the ABRR relation in the case of simple Lie algebras. The proof in the case of quantum groups is analogous but technically more challenging, and is given in Appendix B.

Let $C$ be the quadratic Casimir operator in the center of the universal enveloping algebra $U \mathfrak{g}$ :

$$
C=\sum_{i} x_{i}^{2}+2 \rho+2 \sum_{\alpha \in \Delta^{+}} e_{-\alpha} e_{\alpha}
$$

Then $C$ acts on any highest weight representation of $\mathfrak{g}$ of highest weight $\lambda$ by the scalar $(\lambda, \lambda+2 \rho)$. Now let $V, W$ be two finite-dimensional $\mathfrak{g}$-modules and let $v \in V, w \in W$ be two homogeneous elements of weight $\operatorname{wt}(v)$ and $\operatorname{wt}(w)$. We compute the quantity

$$
F(\lambda)=\left\langle v_{\lambda-\operatorname{wt}(v)-\operatorname{wt}(w)}^{*}, \Phi_{\lambda-\mathrm{wt}(v)}^{w}(C \otimes 1) \Phi_{\lambda}^{v} v_{\lambda}\right\rangle
$$

in two different ways. On one hand we have

$$
F=(\lambda-\operatorname{wt}(v), \lambda-\operatorname{wt}(v)+2 \rho) J(\lambda)(w \otimes v) .
$$

On the other hand,

$$
\begin{aligned}
F=\left\langle v_{\lambda-\operatorname{wt}(v)-\operatorname{wt}(w)}^{*},\{\right. & 2\left(\left(e_{-\alpha} e_{\alpha}\right)_{1}+\left(e_{-\alpha} e_{\alpha}\right)_{2}+\left(e_{\alpha} \otimes e_{-\alpha}\right)_{12}+\left(e_{-\alpha} \otimes e_{\alpha}\right)_{12}+\rho_{1}+\rho_{2}\right) \\
+ & \left.\left.\sum_{i}\left(x_{i}^{2}\right)_{1}+\left(x_{i}^{2}\right)_{2}+2\left(x_{i} \otimes x_{i}\right)_{12}\right\} \Phi_{\lambda-\operatorname{wt}(v)}^{w} \Phi_{\lambda}^{v} v_{\lambda}\right\rangle
\end{aligned}
$$

Since $v_{\lambda-\operatorname{wt}(v)-\operatorname{wt}(w)}^{*}$ is a highest weight vector, it is clear that

$\left\langle v_{\lambda-\operatorname{wt}(v)-\operatorname{wt}(w)}^{*},\left(e_{-\alpha} e_{\alpha}\right)_{1} \Phi_{\lambda-\operatorname{wt}(v)}^{w} \Phi_{\lambda}^{v} v_{\lambda}\right\rangle=\left\langle v_{\lambda-\operatorname{wt}(v)-\operatorname{wt}(w)}^{*},\left(e_{-\alpha} \otimes e_{\alpha}\right)_{12} \Phi_{\lambda-\operatorname{wt}(v)}^{w} \Phi_{\lambda}^{v} v_{\lambda}\right\rangle=0$.

Moreover, by the intertwining property again, we have

$$
\begin{aligned}
\left(e_{\alpha} \otimes e_{-\alpha}\right)_{12} \Phi_{\lambda-\mathrm{wt}(v)}^{w} \Phi_{\lambda}^{v} v_{\lambda}= & -\left(e_{-\alpha} e_{\alpha}\right)_{2}-\left(e_{-\alpha} \otimes e_{\alpha}\right)_{23} \Phi_{\lambda-\mathrm{wt}(v)}^{w} \Phi_{\lambda}^{v} v_{\lambda} \\
\left(\rho_{1}+\rho_{2}\right) \Phi_{\lambda-\mathrm{wt}(v)}^{w} \Phi_{\lambda}^{v} v_{\lambda}= & -\rho_{3} \Phi_{\lambda-\mathrm{wt}(v)}^{w} \Phi_{\lambda}^{v} v_{\lambda}+\Phi_{\lambda-\mathrm{wt}(v)}^{w} \Phi_{\lambda}^{v} \rho v_{\lambda} \\
\left(\left(x_{i}^{2}\right)_{1}+\left(x_{i}^{2}\right)_{2}+2\left(x_{i} \otimes x_{i}\right)_{12}\right) \Phi_{\lambda-\mathrm{wt}(v)}^{w} \Phi_{\lambda}^{v} v_{\lambda}= & \left.-\left(2\left(x_{i} \otimes x_{i}\right)\right)_{13}+2\left(x_{i} \otimes x_{i}\right)_{23}+\left(x_{i}^{2}\right)_{3}\right) \Phi_{\lambda-\operatorname{wt}(v)}^{w} \Phi_{\lambda}^{v} v_{\lambda} \\
& +\Phi_{\lambda-\operatorname{wt}(v)}^{w} \Phi_{\lambda}^{v} x_{i}^{2} v_{\lambda} \\
= & \Phi_{\lambda-\operatorname{wt}(v)}^{w} \Phi_{\lambda}^{v} x_{i}^{2} v_{\lambda}+\left(\left(x_{i}^{2}\right)_{3}-2 \lambda_{3}\right) \Phi_{\lambda-\operatorname{wt}(v)}^{w} \Phi_{\lambda}^{v} v_{\lambda} .
\end{aligned}
$$

Summing up these equations, we finally obtain

$$
F=\left(-2 \rho_{\mid V}+2(\rho, \lambda)+\left(\sum_{i} x_{i}^{2}\right)_{\mid V}-2 \lambda_{\mid V}+\lambda^{2}-2 \sum_{\alpha \in \Delta^{+}} e_{-\alpha} \otimes e_{\alpha}\right) J(\lambda)
$$


Combining (8.3) and (8.4) yields

$$
\left(\sum_{i} x_{i}^{2}-2(\lambda+\rho)\right)_{\mid V} J(\lambda)-\left(\operatorname{wt}(v)^{2}-2(\lambda+\rho, \operatorname{wt}(v))\right) J(\lambda)=-2\left(\sum_{\alpha \in \Delta^{+}} e_{-\alpha} \otimes e_{\alpha}\right) J(\lambda)
$$

which is equivalent to 8.2 .

Example. Let us use the recursive procedure in the proof above to compute $J(\lambda)$ for $U\left(s l_{2}\right)$. Setting $J(\lambda)=1+\sum_{n \geq 1} J^{(n)}(\lambda)$ where $J^{(n)}(\lambda) \in$ $U^{\prime}\left(\mathfrak{b}_{-}\right)[-2 n] \otimes U^{\prime}\left(\mathfrak{b}_{+}\right)[2 n]$, the ABRR equation reads

$$
\frac{1}{2}\left[1 \otimes\left((\lambda+1) h-\frac{h^{2}}{2}\right), J-1\right]=-(f \otimes e) J,
$$

which gives the recurrence relation

$$
1 \otimes\left((\lambda+1) n-n h+n^{2}\right) J^{(n)}=(-f \otimes e) J^{(n-1)} .
$$

Hence

$$
J^{(n)}(\lambda)=\frac{(-1)^{n}}{n !} f^{n} \otimes(\lambda-h+2)^{-1} \ldots(\lambda-h+n+1)^{-1} e^{n} .
$$

This formula and its quantum analogue were obtained in the pioneering paper [BBB], which was a motivation to the authors of ABRR].

8.2. Classical limits of fusion and exchange matrices. The ABRR relations can also be used to derive the classical limits of the fusion (and thus of the exchange) matrices. Setting $q=e^{-\gamma / 2}$, rescaling $\lambda \mapsto \frac{\lambda}{\gamma}$ and considering the limit $\gamma \rightarrow 0$ yields the following classical version of the ABRR equation:

$$
\operatorname{Ad}\left(1 \otimes e^{-\lambda}\right) j(\lambda)-j(\lambda)=\sum_{\alpha \in \Delta^{+}} e_{-\alpha} \otimes e_{\alpha}
$$

which admits the unique lower triangular solution

$$
j(\lambda)=-\sum_{\alpha \in \Delta^{+}} \frac{e_{-\alpha} \otimes e_{\alpha}}{1-e^{-(\alpha, \lambda)}} .
$$

From this we deduce

$r(\lambda)=r^{21}+j(\lambda)-j^{21}(\lambda)=\frac{1}{2} \Omega+\frac{1}{2} \sum_{\alpha>0} \operatorname{cotanh}\left(\frac{1}{2}\langle\alpha, \lambda\rangle\right)\left(e_{\alpha} \otimes e_{-\alpha}-e_{-\alpha} \otimes e_{\alpha}\right)$

which is consistent with Proposition 3.3.

The case of a simple Lie algebra $\mathfrak{g}$ is completely analogous; the classical version of the ABRR equation is

$$
[j(\lambda), \lambda \otimes 1]=\sum_{\alpha \in \Delta^{+}} e_{-\alpha} \otimes e_{\alpha},
$$

which admits the unique solution

$$
j(\lambda)=-\sum_{\alpha \in \Delta^{+}} \frac{e_{-\alpha} \otimes e_{\alpha}}{(\lambda, \alpha)} .
$$

This yields Corollary 3.1. 


\section{Transfer matrices and Generalized Macdonald- Ruijsenaars equations}

9.1. Transfer matrices. We first recall the well-known transfer matrix construction.

Let $A$ be a Hopf algebra with a commutative Grothendieck ring, and let $\mathcal{R} \in A \otimes A$ be an element such that $(\Delta \otimes 1)(\mathcal{R})=\mathcal{R}^{13} \mathcal{R}^{23}$. A basic example is: $A$ is quasitriangular, $\mathcal{R}$ is its universal $\mathrm{R}$-matrix.

For any finite-dimensional representation $\pi_{V}: A \rightarrow$ End $V$ of $A$, set

$$
T_{V}=\operatorname{Tr}_{\mid V}\left(\pi_{V} \otimes 1\right)(\mathcal{R}) \in A .
$$

These elements are called transfer matrices.

Lemma 9.1. For any finite-dimensional $A$-modules $V, W$ we have $T_{V} T_{W}=$ $T_{V \otimes W}=T_{W} T_{V}$.

Proof. By definition we have

$$
\left(\pi_{V \otimes W} \otimes 1\right) \mathcal{R}=\left(\pi_{V} \otimes \pi_{W} \otimes 1\right)(\Delta \otimes 1) \mathcal{R}=\left(\pi_{V} \otimes 1\right) \mathcal{R}_{13}\left(\pi_{W} \otimes 1\right) \mathcal{R}_{23},
$$

which implies the first equality. The second equality follows from the commutativity of the Grothendieck ring.

The transfer matrix construction gives rise to interesting examples of quantum integrable systems which arise in statistical mechanics. For example, if $A$ is the quantum affine algebra or the elliptic algebra, one gets transfer matrices of the 6 -vertex and 8-vertex models, respectively.

We adapt the notion of transfer matrices in our dynamical setting in the following way. Let $\mathfrak{g}$ be a simple Lie algebra and let $U_{q}(\mathfrak{g})$ be the associated quantum group. For any two finite-dimensional $U_{q}(\mathfrak{g})$-modules $V$ and $W$ let $R_{V W}(\lambda)$ be the exchange matrix. It is more convenient to work with the shifted exchange matrix $\mathbb{R}(\lambda)=R(-\lambda-\rho)$.

Let $\mathcal{F}_{V}$ be the space of $V[0]$-valued meromorphic functions on $\mathfrak{h}^{*}$. For $\nu \in \mathfrak{h}^{*}$ let $T_{\nu} \in \operatorname{End}\left(\mathcal{F}_{V}\right)$ be the shift operator $\left(T_{\nu} f\right)(\lambda)=f(\lambda+\nu)$. As pointed out in FV3, the role of the transfer matrix is played by the following difference operator

$$
\mathcal{D}_{W}^{V}=\sum_{\nu} \operatorname{Tr}_{\mid W[\nu]}\left(\mathbb{R}_{W V}(\lambda)\right) T_{\nu}
$$

It follows from the dynamical 2-cocycle condition for fusion matrices (see Proposition 2.3) that for any $U_{q}(\mathfrak{g})$-modules $U, V, W$ we have

$$
\mathcal{D}_{V \otimes W}^{U}=\mathcal{D}_{V}^{U} \mathcal{D}_{W}^{U}=\mathcal{D}_{W}^{U} \mathcal{D}_{V}^{U} .
$$

Hence $\left\{\mathcal{D}_{W}^{U}\right\}$ span a commuting family of difference operators acting on $\mathcal{F}_{U}$. 
9.2. Weighted trace functions. Let $V$ be a finite-dimensional $U_{q}(\mathfrak{g})$-module. Recall that, for any homogeneous vector $v \in V[\nu]$ and for generic $\mu \in \mathfrak{h}^{*}$ there exists a unique intertwiner $\Phi_{\mu}^{v}: M_{\mu} \rightarrow M_{\mu-\nu} \otimes V$ such that $\left\langle v_{\mu-\nu}^{*}, \Phi_{\mu}^{v} v_{\mu}\right\rangle=v$. Set

$$
\Phi_{\mu}^{V}=\sum_{v \in \mathcal{B}} \Phi_{\mu}^{v} \otimes v^{*} \in \operatorname{Hom}_{\mathbb{C}}\left(M_{\mu}, \bigoplus_{\nu} M_{\mu-\nu} \otimes V[\nu] \otimes V^{*}[-\nu]\right),
$$

where $\mathcal{B}$ is any homogeneous basis of $V$. Consider the weighted trace function

$$
\Psi_{V}(\lambda, \mu)=\operatorname{Tr}\left(\Phi_{\mu}^{V} q^{2 \lambda}\right) \in V[0] \otimes V^{*}[0]
$$

where $q^{2 \lambda}$ acts on any $\mathfrak{h}$-semisimple $U_{q}(\mathfrak{g})$-module $U$ by $q_{\mid U[\nu]}^{2 \lambda}=q^{2(\lambda, \nu)} I d$. It can be shown that $\Psi_{V} \in V[0] \otimes V^{*}[0] \otimes q^{2(\lambda, \mu)} \mathbb{C}\left(q^{\lambda}\right) \otimes \mathbb{C}\left(q^{\mu}\right)$. Let

$$
\delta_{q}(\lambda)=\left(\operatorname{Tr}_{\mid M_{-\rho}}\left(q^{2 \lambda}\right)\right)^{-1}=q^{-2(\lambda, \rho)} \prod_{\alpha>0}\left(1-q^{-2(\lambda, \alpha)}\right)
$$

be the Weyl denominator, and set

$$
Q(\lambda)=m^{o p}\left(1 \otimes S^{-1}\right)(J(-\lambda-\rho)),
$$

where $m^{o p}: U_{q}(\mathfrak{g}) \otimes U_{q}(\mathfrak{g}) \rightarrow U_{q}(\mathfrak{g}), a \otimes b \mapsto b a$ and where $J(\lambda)$ is the universal fusion matrix. It can be shown that $Q(\lambda)$ is invertible. Finally, set

$$
F_{V}(\lambda, \mu)=Q^{-1}(\mu)_{\mid V^{*}} \Psi_{V}(\lambda,-\mu-\rho) \delta_{q}(\lambda) .
$$

Theorem 9.1 ([EV4], The Macdonald-Ruijsenaars equations). For any two finite-dimensional $U_{q}(\mathfrak{g})$-modules, we have

$$
\mathcal{D}_{W}^{\lambda, V} F_{V}(\lambda, \mu)=\chi_{W}\left(q^{-2 \mu}\right) F_{V}(\lambda, \mu)
$$

where $\chi_{W}\left(q^{x}\right)=\sum \operatorname{dim} W[\nu] q^{(\nu, x)}$ is the character of $W$.

Theorem 9.2 ([EV4], The dual Macdonald-Ruijsenaars equations). For any two finite-dimensional $U_{q}(\mathfrak{g})$-modules, we have

$$
\mathcal{D}_{W}^{\mu, V^{*}} F_{V}(\lambda, \mu)=\chi_{W}\left(q^{-2 \lambda}\right) F_{V}(\lambda, \mu)
$$

In the above, we add a superscript to $\mathcal{D}$ to specify on which variable the difference operators act. Thus, in Theorem 9.1, $\mathcal{D}_{W}^{V}$ acts on functions of the variable $\lambda$ in the component $V[0]$, and in Theorem 9.2, $\mathcal{D}_{W}^{V^{*}}$ acts on functions in the variable $\mu$ in the component $V^{*}[0]$.

From Theorems 9.1 and 9.2 it is not difficult to deduce the following result:

Theorem 9.3 ([EV4], The symmetry identity). For any finite-dimensional $U_{q}(\mathfrak{g})$-module we have

$$
F_{V}(\lambda, \mu)=F_{V^{*}}^{*}(\mu, \lambda),
$$

where $*: V[0] \otimes V^{*}[0] \rightarrow V^{*}[0] \otimes V[0]$ is the permutation. 
9.3. Relation to Macdonald theory. Let us now restrict ourselves to the case of $\mathfrak{g}=s l_{n}$, and let $V$ be the q-analogue of the representation $S^{m n} \mathbb{C}^{n}$. The zero-weight subspace of this representation is 1-dimensional, so the function $\Psi_{V}$ can be regarded as a scalar function. We will denote this scalar function by $\Psi_{m}(q, \lambda, \mu)$.

Recall the definition of Macdonald operators Ma, EK1. They are operators on the space of functions $f\left(\lambda_{1}, \ldots, \lambda_{n}\right)$ which are invariant under simultaneous shifting of the variables, $\lambda_{i} \rightarrow \lambda_{i}+c$, and have the form

$$
M_{r}=\sum_{I \subset\{1, \ldots, n\}:|I|=r}\left(\prod_{i \in I, j \notin I} \frac{t q^{2 \lambda_{i}}-t^{-1} q^{2 \lambda_{j}}}{q^{2 \lambda_{i}}-q^{2 \lambda_{j}}}\right) T_{I},
$$

where $T_{I} \lambda_{j}=\lambda_{j}$ if $j \notin I$ and $T_{I} \lambda_{j}=\lambda_{j}+1$ if $j \in I$. Here $q, t$ are parameters. We will assume that $t=q^{m+1}$, where $m$ is a nonnegative integer.

It is known $\mathrm{Ma}$ that the operators $M_{r}$ commute. From this it can be deduced that for a generic $\mu=\left(\mu_{1}, \ldots, \mu_{n}\right), \sum \mu_{i}=0$, there exists a unique power series $f_{m 0}(q, \lambda, \mu) \in \mathbb{C}\left[\left[q^{\lambda_{2}-\lambda_{1}}, \ldots, q^{\lambda_{n}-\lambda_{n-1}}\right]\right]$ such that the series $f_{m}(q, \lambda, \mu):=$ $q^{2(\lambda, \mu-m \rho)} f_{m 0}(q, \lambda, \mu)$ satisfies difference equations

$$
M_{r} f_{m}(q, \lambda, \mu)=\left(\sum_{I \subset\{1, \ldots, n\}:|I|=r} q^{2 \sum_{i \in I}(\mu+\rho)_{i}}\right) f_{m}(q, \lambda, \mu) .
$$

Remark. The series $f_{m 0}$ is convergent to an analytic (in fact, a trigonometric) function.

The following theorem is contained in [EK1].

Theorem 9.4 ([EK1], Theorem 5). One has

$$
f_{m}(q, \lambda, \mu)=\gamma_{m}(q, \lambda)^{-1} \Psi_{m}\left(q^{-1},-\lambda, \mu\right)
$$

where

$$
\gamma_{m}(q, \lambda):=\prod_{i=1}^{m} \prod_{l<j}\left(q^{\lambda_{l}-\lambda_{j}}-q^{2 i} q^{\lambda_{j}-\lambda_{l}}\right) .
$$

Let $\mathcal{D}_{W}\left(q^{-1},-\lambda\right)$ denote the difference operator, obtained from the operator $\mathcal{D}_{W}$ defined in Section 1 by the transformation $q \rightarrow q^{-1}$ and the change of coordinates $\lambda \rightarrow-\lambda$. Let $\Lambda^{r} \mathbb{C}^{n}$ denote the q-analog of the r-th fundamental representation of $s l_{n}$.

\section{Corollary 9.1.}

$$
\mathcal{D}_{\Lambda^{r} \mathbb{C}^{n}}\left(q^{-1},-\lambda\right)=\delta_{q}(\lambda) \gamma_{m}(q, \lambda) \circ M_{r} \circ \gamma_{m}(q, \lambda)^{-1} \delta_{q}(\lambda)^{-1} .
$$

Proof. This follows from Theorem 9.4 and Theorem 9.1.

Remark. In the theorems of this section, Verma modules $M_{\mu}$ can be replaced with finite dimensional irreducible modules $L_{\mu}$ with sufficiently large highest weight, and one can prove analogs of these theorems in this situation (in the same way as for Verma modules). In particular, one may set $\hat{\Psi}_{m}(q, \lambda, \mu)=$ $\operatorname{Tr}\left(\hat{\Phi}_{\mu}^{V} q^{2 \lambda}\right)$, where $\hat{\Phi}_{\mu}^{V}: L_{\mu} \rightarrow L_{\mu} \otimes V \otimes V^{*}[0]$ is the intertwiner with highest coefficient 1 (Such an operator exists iff $\mu-m \rho \geq 0$, see [EK1). Then one can 
show analogously to Theorem 9.1 (see [EK1) that the function $\hat{f}_{m}(q, \lambda, \mu):=$ $\gamma_{m}(q, \lambda)^{-1} \hat{\Psi}_{m}\left(q^{-1},-\lambda, \mu+m \rho\right)$ is the Macdonald polynomial $P_{\mu}\left(q, t, q^{2 \lambda}\right)$ with highest weight $\mu$ ( $\mu$ is a dominant integral weight). In this case, Theorem 9.1 says that Macdonald's polynomials are eigenfunctions of Macdonald's operators, Theorem 9.2 gives recursive relations for Macdonald's polynomials with respect to the weight (for $\operatorname{sl}(2)$ - the usual 3-term relation for orthogonal polynomials), and Theorem 9.3 is the Macdonald symmetry identity (see [Ma]).

\section{Appendix A: Classical dynamical r-matrices on a simple Lie algebra $\mathfrak{g}$ with respect to $\mathfrak{l} \subset \mathfrak{h}$.}

A.1. Let $\mathfrak{g}$ be a simple complex Lie algebra and $\mathfrak{l}$ a commutative subalgebra of $\mathfrak{g}$ consisting of semisimple elements. Then $\mathfrak{l} \subset \mathfrak{h}$ for some Cartan subalgebra $\mathfrak{h}$. We keep the notations of Section 1. In this appendix, we give a classification of all classical dynamical r-matrices $\mathfrak{l}^{*} \rightarrow(\mathfrak{g} \otimes \mathfrak{g})^{\mathfrak{l}}$ with coupling constant 1 . Note that we can suppose without loss of generality that the restriction of $\langle$,$\rangle to \mathfrak{l}$ is nondegenerate. Indeed, given a dynamical r-matrix $r: \mathfrak{l}^{*} \rightarrow(\mathfrak{g} \otimes \mathfrak{g})^{\mathfrak{l}}$, we can always replace $\mathfrak{l}$ by the largest subalgebra of $\mathfrak{h}$ under which $r$ is invariant, and this subalgebra is real.

A.2. Gauge transformations. Let $\Omega^{\prime} \in \mathfrak{l}^{\perp} \otimes \mathfrak{l}^{\perp}$ (where the orthogonal complement is in $\mathfrak{g}$ ) be the inverse (Casimir) element to the form $\langle$,$\rangle . If$ $r(\lambda)=\frac{1}{2} \Omega+(\varphi(\lambda) \otimes 1) \Omega^{\prime}, \varphi: \mathfrak{l}^{*} \rightarrow \operatorname{End}_{\mathfrak{l}}\left(\mathfrak{l}^{\perp}\right)$ is a meromorphic function with values in $(\mathfrak{g} \otimes \mathfrak{g})^{\mathfrak{l}}$, and if $f: \mathfrak{l}^{*} \rightarrow \mathfrak{h}$ is any meromorphic function, set

$$
r^{f}(\lambda)=\frac{1}{2} \Omega+\left(e^{-a d f(\lambda)} \varphi(\lambda) e^{a d f(\lambda)} \otimes 1\right) \Omega^{\prime}
$$

Lemma 10.1. The transformations $r(\lambda) \mapsto r^{f}(\lambda)$ preserve the set of classical dynamical r-matrices with coupling constant 1.

Two r-matrices which can be obtained one from the other by such a transformation are called gauge-equivalent.

A.3. Classification of dynamical $\mathbf{r}$-matrices. Let $\mathfrak{h}$ be a Cartan subalgebra of $\mathfrak{g}$, and let $\Pi \subset \mathfrak{h}^{*}$ be a system of simple roots in $\Delta$. Let $\mathfrak{h}_{0} \subset \mathfrak{h}$ be the orthogonal complement of $\mathfrak{l}$ in $\mathfrak{h}$.

Definition. A generalized Belavin-Drinfeld triple is a triple $\left(\Gamma_{1}, \Gamma_{2}, \tau\right)$ where $\Gamma_{1}, \Gamma_{2} \subset \Pi$, and where $\tau: \Gamma_{1} \stackrel{\sim}{\rightarrow} \Gamma_{2}$ is a norm-preserving isomorphism.

Given a generalized Belavin-Drinfeld triple $\left(\Gamma_{1}, \Gamma_{2}, \tau\right)$, we extend linearly the map $\tau$ to a norm-preserving bijection $\left\langle\Gamma_{1}\right\rangle \rightarrow\left\langle\Gamma_{2}\right\rangle$, where $\left\langle\Gamma_{1}\right\rangle$ (resp. $\left\langle\Gamma_{2}\right\rangle$ ) is the set of roots $\alpha \in \Delta$ which are linear combinations of simple roots from $\Gamma_{1}$ (resp. from $\Gamma_{2}$ ).

We will say that a generalized Belavin-Drinfeld triple $\left(\Gamma_{1}, \Gamma_{2}, \tau\right)$ is $\mathfrak{l}$-admissible 
if $\tau(\alpha)-\alpha \in \mathfrak{l}^{\perp}$ for all $\alpha \in \Gamma_{1}$, if $\tau$ satisfies the following condition: for every cycle $\alpha \mapsto \tau(\alpha) \mapsto \ldots \mapsto \tau^{r}(\alpha)=\alpha$ we have $\alpha+\tau(\alpha)+\ldots+\tau^{r-1} \alpha \in \mathfrak{l}$.

If $\left(\Gamma_{1}, \Gamma_{2}, \tau\right)$ is a generalized Belavin-Drinfeld triple, let $\mathfrak{g}_{\Gamma_{1}}, \mathfrak{g}_{\Gamma_{2}}$ be the subalgebras generated by $e_{\alpha}, f_{\alpha} \alpha \in \Gamma_{1}$ (resp. generated by $e_{\alpha}, f_{\alpha} \alpha \in \Gamma_{2}$ ). The map $\tau: e_{\alpha} \mapsto e_{\tau(\alpha)}, \alpha \in \Gamma_{1}$ extends to an isomorphism $\tau: \mathfrak{g}_{\Gamma_{1}} \stackrel{\sim}{\rightarrow} \mathfrak{g}_{\Gamma_{2}}$. Finally, define an operator $K: \mathfrak{l}^{*} \rightarrow \operatorname{Hom}\left(\mathfrak{g}_{\Gamma_{1}}, \mathfrak{g}\right)$ by

$$
K(\lambda) e_{\alpha}=\sum_{n>0} e^{-n(\alpha, \lambda)} \tau^{n}\left(e_{\alpha}\right) .
$$

Notice that this sum is finite if $\tau$ acts nilpotently on $\alpha$.

Theorem $10.1([\mathbf{S}])$. Let $\left(\Gamma_{1}, \Gamma_{2}, \tau\right)$ be an $\mathfrak{l}$-admissible generalized BelavinDrinfeld triple.

(i) The equation

$$
((\alpha-\tau(\alpha)) \otimes 1) r_{0}=\frac{1}{2}((\tau(\alpha)+\alpha) \otimes 1) \Omega_{\mathfrak{h}_{0}},
$$

where $\Omega_{\mathfrak{h}_{0}} \subset \mathfrak{h}_{0} \otimes \mathfrak{h}_{0}$ is the inverse element to the form $\langle$,$\rangle , has solutions$ $r_{0} \in \Lambda^{2} \mathfrak{h}_{0}$.

(ii) Let $r_{0} \in \Lambda^{2} \mathfrak{h}_{0}$ satisfy the equation from (i). Then

$$
r(\lambda)=\frac{1}{2} \Omega+r_{0}+\sum_{\substack{\alpha \in \Delta^{+} \\ e_{\alpha} \in \mathfrak{g}_{\Gamma_{1}}}} K(\lambda) e_{\alpha} \wedge f_{\alpha}+\sum_{\alpha \in \Delta^{+}} \frac{1}{2} e_{\alpha} \wedge f_{\alpha}
$$

is a classical dynamical r-matrix. Conversely, any classical dynamical r-matrix $r: \mathfrak{l}^{*} \rightarrow(\mathfrak{g} \otimes \mathfrak{g})^{\mathfrak{l}}$ with coupling constant 1 is gauge equivalent to one of the above form, for suitable choices of Cartan subalgebra $\mathfrak{h}$ containing $\mathfrak{l}$, polarization of $\mathfrak{g}$ and $\mathfrak{l}$-admissible generalized Belavin-Drinfeld triple.

Proof. Let us prove statement (i); statement (ii) is proved in [\$] . Let $\mathfrak{l}_{\max }$ be the Lie algebra of all $x \in \mathfrak{h}$ such that $(\alpha-\tau(\alpha), x)=0$, and let $\mathfrak{p}$ be the orthogonal complement of $\mathfrak{l}$ in $\mathfrak{l}_{\max }$. Then we have an orthogonal direct sum decomposition $\mathfrak{h}_{0}=\mathfrak{p} \oplus \mathfrak{l}_{\max }^{\perp}$. Let us regard $r_{0}$ as a bilinear form on $\mathfrak{h}$ (via the standard inner product). The equation from (i) determines $r_{0}(x, y)$ where $x \in \mathfrak{l}_{\max }^{\perp}$, and $y$ is arbitrary. To check that $r_{0}$ can be extended to a skew symmetric form, it suffices to check that it is skew-symmetric on $\mathfrak{l}_{\text {max }}^{\perp}$. But using the equation from (i) we find that

$$
r_{0}(\alpha-\tau(\alpha), \beta-\tau(\beta))=1 / 2(\alpha+\tau(\alpha), \beta-\tau(\beta))=\frac{1}{2}((\beta, \tau(\alpha))-(\alpha, \tau(\beta)))
$$

(we use that $\tau$ preseves $($,$) ), which is obviously skew symmetric.$

Remarks. 1. This classification is very similar in spirit to the classification of classical r-matrices $r \in \mathfrak{g} \otimes \mathfrak{g}$ satisfying $r+r^{21}=\Omega$ (quasitriangular structures) obtained by Belavin and Drinfeld, and reduces to it for $\mathfrak{l}=0$ (see $[B D]$ ).

2. When $\mathfrak{l}=\mathfrak{h}$ one recovers the classification result Theorem 4.2: the only $\mathfrak{h}$-graded generalized Belavin-Drinfeld triples are of the form $\Gamma_{1}=\Gamma_{2}, \tau=I d$, and in this case the r-matrix (10.1) corresponds to $r_{X}^{1}(\lambda)$, with $X=\Gamma_{1}$.

3. Theorem 10.1 is proved in $[S \mid$ under the additional assumption that $\mathfrak{l}$ contains a regular semisimple element. However, the proof easily extends to the present situation. 


\section{Appendix B: Proof of the ABRR relation for $U_{q}(\mathfrak{g})$}

We keep the notations of Section 8. Recall the Drinfeld construction of the quantum Casimir element of $U_{q}(\mathfrak{g})$. Let $\mathcal{R}$ be the universal R-matrix for $U_{q}(\mathfrak{g})$. Let us write $\mathcal{R}=\sum_{i} a_{i} \otimes b_{i}$ and set $u=\sum S\left(b_{i}\right) a_{i}$. Then $u=q^{2 \rho} z$ where $z$ is a central element in a completion of $U_{q}(\mathfrak{g})$, which is called the quantum Casimir element. Moreover, for any $\mu \in \mathfrak{h}^{*}$ we have

$$
u v_{\mu}=q^{-\sum_{i} x_{i}^{2}} v_{\mu}=q^{-(\mu, \mu)} v_{\mu}
$$

hence $z v_{\mu}=q^{-(\mu, \mu+2 \rho)} v_{\mu}$ and $u_{\mid M_{\mu}}=q^{-(\mu, \mu+2 \rho)} q^{2 \rho}$.

Now let $V$ and $W$ be two finite-dimensional $U_{q}(\mathfrak{g})$-modules, $v \in V$ and $w \in$ $W$ homogeneous vectors of weight $\mu_{v}$ and $\mu_{w}$ respectively, and consider the expectation value

$$
X_{v w}(\mu)=\left\langle v_{\mu-\mu_{v}-\mu_{w}}^{*}, \Phi_{\mu-\mu_{v}}^{w} u_{\mid M_{\mu-\mu_{v}}} \Phi_{\mu}^{v} v_{\mu}\right\rangle .
$$

We will compute $X_{v w}(\mu)$ in two different ways. On one hand,

$$
\begin{aligned}
X_{v w}(\mu) & =\left\langle v_{\mu-\mu_{v}-\mu_{w}}^{*}, \Phi_{\mu-\mu_{v}}^{w}\left(q^{2 \rho} z\right)_{\mid M_{\mu-\mu_{v}}} \Phi_{\mu}^{v} v_{\mu}\right\rangle \\
& =\left\langle v_{\mu-\mu_{v}-\mu_{w}}^{*}, \Phi_{\mu-\mu_{v}}^{w} z_{\mid M_{\mu-\mu_{v}}} q_{\mid V}^{-2 \rho} \Phi_{\mu}^{v} q^{2 \rho} v_{\mu}\right\rangle \\
& =q^{-\left(\mu-\mu_{v}, \mu-\mu_{v}+2 \rho\right)} q_{\mid V}^{2 \rho} q^{(2 \rho, \mu)} J(\mu)(w \otimes v) \\
& =q^{-\left(\mu-\mu_{v}, \mu-\mu_{v}\right)+2\left(\rho, \mu_{v}\right)} q_{\mid V}^{-2 \rho} J(\mu)(w \otimes v)
\end{aligned}
$$

On the other hand, we have $\left(1 \otimes \Delta^{o p}\right) \mathcal{R}=\mathcal{R}^{12} \mathcal{R}^{13}$ hence, by the intertwining property,

$$
(1 \otimes \Delta)(1 \otimes S) \mathcal{R}=\sum_{i j} a_{i} a_{j} \otimes S\left(b_{i}\right) \otimes S\left(b_{j}\right)
$$

Thus

$$
X_{v w}(\mu)=\sum_{i, j} S\left(b_{j}\right)_{\mid W}\left\langle v_{\mu-\mu_{v}-\mu_{w}}^{*}, S\left(b_{i}\right)_{\mid M_{\mu-\mu_{v}-\mu_{w}}} \Phi_{\mu-\mu_{v}}^{w} a_{i} a_{j \mid M_{\mu-\mu_{v}}} \Phi_{\mu}^{v} v_{\mu}\right\rangle .
$$

Now, since $v_{\mu-\mu_{v}-\mu_{w}}^{*}$ is a lowest weight vector and since

$$
\mathcal{R} \in\left(1+U_{q}^{\prime}\left(\mathfrak{b}_{-}\right) \otimes U_{q}^{\prime}\left(\mathfrak{b}_{+}\right)\right) q^{\sum_{i} x_{i} \otimes x_{i}}
$$

equation (11.1) reduces to

$$
\begin{aligned}
& X_{v w}(\mu)=\sum_{j} S\left(b_{j}\right)_{\mid W}\left\langle v_{\mu-\mu_{v}-\mu_{w}}^{*}, q_{\mid M_{\mu-\mu_{v}-\mu_{w}}}^{-\sum_{\mid M_{\mu-\mu_{v}-\mu_{w}} \otimes W}^{2}} q_{\mu-\mu_{v}}^{-\sum x_{i} \otimes x_{i}} a_{j \mid M_{\mu-\mu_{v}}} \Phi_{\mu}^{v} v_{\mu}\right\rangle \\
& =q^{-\left(\mu-\mu_{v}-\mu_{w}\right)^{2}} \sum_{j}\left(S\left(b_{j}\right) q^{-\mu+\mu_{v}+\mu_{w}}\right)_{\mid W}\left\langle v_{\mu-\mu_{v}-\mu_{w}}^{*} \Phi_{\mu-\mu_{v}}^{w} a_{j \mid M_{\mu-\mu_{v}}} \Phi_{\mu}^{v} v_{\mu}\right\rangle
\end{aligned}
$$

Using the relation

$$
(\Delta \otimes S) \mathcal{R}=\sum a_{l} \otimes a_{k} \otimes S\left(b_{k}\right) S\left(b_{l}\right)
$$


we have

$$
\sum_{j} S\left(b_{j}\right)_{\mid W} \otimes \Phi_{\mu}^{v} a_{j \mid M_{\mu}}=\left(\sum_{k, l}\left(S\left(b_{k}\right) S\left(b_{l}\right)\right)_{\mid W} \otimes a_{l \mid M_{\mu-\mu_{v}}} \otimes a_{k \mid V}\right) \Phi_{\mu}^{v},
$$

i.e

$$
\left(\sum S\left(b_{l}\right)_{\mid W} \otimes a_{l \mid M_{\mu-\mu_{v}}}\right) \Phi_{\mu}^{v}=\left(\sum S\left(b_{k}\right)_{\mid W} \otimes a_{k \mid V}\right)^{-1} \sum S\left(b_{j}\right)_{\mid W} \otimes \Phi_{\mu}^{v} a_{j \mid M_{\mu}} .
$$

Substitution of this in (11.2) gives

$$
X_{v w}(\mu)=q^{-\left(\mu-\mu_{v}-\mu_{w}\right)^{2}}\left(\sum S\left(b_{k}\right)_{\mid W} \otimes a_{k \mid V}\right)^{-1} q_{\mid W}^{-2 \mu+\mu_{v}+\mu_{w}} J(\mu)(w \otimes v)
$$

Claim: we have $\left(\sum S\left(b_{k}\right) \otimes a_{k}\right)^{-1}=\left(1 \otimes q^{2 \rho}\right) \mathcal{R}\left(1 \otimes q^{-2 \rho}\right)$.

Proof. We have $S^{2}(a)=q^{2 \rho} a q^{-2 \rho}$ for any $a \in U_{q}(\mathfrak{g})$. Hence $\sum a_{k} \otimes S\left(b_{k}\right)=$ $\left(1 \otimes q^{2 \rho}\right)\left(1 \otimes S^{-1}\right)\left(1 \otimes q^{-2 \rho}\right)$. The claim now follows from the relation

$\left(1 \otimes S^{-1}\right) \mathcal{R}=\mathcal{R}^{-1}$.

Thus, combining (11.1) and (11.3), we get

$$
\begin{aligned}
q^{-\left(\mu-\mu_{v}, \mu-\mu_{v}+2 \rho\right)} & q_{\mid V}^{-2 \rho} q^{2(\mu, \rho)} J(\mu)(w \otimes v) \\
= & q^{-\left(\mu-\mu_{v}-\mu_{w}\right)^{2}} q_{\mid W}^{2 \rho} \mathcal{R}^{21} q_{\mid W}^{\mu_{v}+\mu_{w}-2(\mu+\rho)} J(\mu)(w \otimes v) .
\end{aligned}
$$

This implies that

$$
\begin{aligned}
q^{-\left(\mu_{v}+\mu_{w}\right)^{2}} & \mathcal{R}^{21} q_{\mid W}^{\mu_{v}+\mu_{w}-2(\mu+\rho)} J(\mu)(w \otimes v) \\
& =q^{\mu^{2}-2\left(\mu, \mu_{v}+\mu_{w}\right)} q_{\mid V}^{-2 \rho} q_{\mid W}^{-2 \rho} q^{2(\mu, \rho)} q^{-\left(\mu-\mu_{v}, \mu-\mu_{v}+2 \rho\right)} J(\mu)(w \otimes v)
\end{aligned}
$$

Using the weight zero property of $\mathcal{R}$, we can rewrite the l.h.s of this last equation as

$$
\mathcal{R}^{21} q_{\mid W \otimes V}^{-\sum x_{i} \otimes x_{i}} q_{\mid V}^{-\sum x_{i}^{2}} q_{\mid W}^{-2(\mu+\rho)} J(\mu)(w \otimes v)
$$

Similarly, using the weight zero property of $J(\mu)$, it is easy to see that the right hand side is equal to

$$
q^{-2\left(\mu+\rho, \mu_{w}\right)} q^{-\mu^{2}} J(\mu)=J(\mu) q_{\mid W}^{-2(\mu+\rho)} q_{\mid V}^{-\sum x_{i}^{2}}(w \otimes v)
$$

The ABRR equation now follows from (11.4), 111.5), 111.6) and the weight zero property of $J(\mu)$.

\section{Review of literature}

In conclusion, we would like to give a brief review of the existing literature on the dynamical Yang-Baxter equations. We would like to make it clear that this list is by no means complete, and contains only some of the basic references which are relevant to this paper. 
The physical paper in which the dynamical Yang-Baxter equation was first considered is GN]; dynamical R-matrices are also discussed in [Fad1, [AF].

The classical dynamical Yang-Baxter equation and examples of its solutions were introduced in $[\mathrm{F}]$. Its geometric interpretation in terms of Poisson groupoids of Weinstein [M] was introduced in [EV1]. Solutions of this equation were studied and classified in [EV1], [S]. The relationship of solutions of this equation to Poisson groupoids and Lie bialgebroids was further explored in [LX] and BK-S]. The relationship of solutions of the classical dynamical Yang-Baxter equation (defined on noncommutative Lie algebras) to equivariant cohomology is discussed in AM. The relationship to integrable systems is discussed in $\mathrm{ABB}$.

Quantum groups associated to a dynamical R-matrix were first introduced in $[\mathbb{E}]$. In the case when the R-matrix is elliptic, they are called elliptic quantum groups. These quantum groups and their representation theory (for the Lie algebras $s l_{n}$ ), as well as their relationship with integrable systems, were systematically studied in [FV1, FV2, EV3]. The papers [EV2, EV3 study the trigonometric versions of these quantum groups (for any simple Lie algebra).

Quantum groupoids were introduced by Maltsiniotis (in the case when base is classical), and by Lu in [Lu] in the general case. The interpretation of dynamical quantum groups as quantum groupoids was first discussed in EV2, EV3, and further studied in [Xu1], [Xu2]. The interpretation of dynamical quantum groups as quasi-Hopf algebras is contained in BBB], and was further developed in Fr, JKOS, ABRR, EF]. The connection between these two interpretation is discussed in Xu1.

Quantum KZB equations (which are not discussed in these notes) were introduced in $[\mathrm{F}]$, and studied in $[$ FTV1, FTV2, MV, FV3, FV4, FV5, FV6, FV7]. Monodromy of quantum KZ equations [FR], which yields dynamical R-matrices of the elliptic quantum groups, is computed in TTV1, TV2].

The theory of traces of intertwining operators for Lie algebras and quantum groups and its applications to the theory of special functions (in particular, Macdonald theory) is developed in [B], [E], E], EK1, EK2, EK3, EK4, K1, K2, K3], EFK], ES1, ES2]. The relationship of this theory with dynamical Rmatrices is studied in [EV4].

\section{References}

[ABB] Avan J., Babelon O., Billey E., The Gervais-Neveu-Felder equation and the quantum Calogero-Moser systems, hep-th/9505091, Comm. Math. Phys., 178, issue 2, (1996) 281-299.

[ABRR] D.Arnaudon, E.Buffenoir, E.Ragoucy, and Ph.Roche, Universal Solutions of quantum dynamical Yang-Baxter equations, Lett. Math. Phys. 44 (1998), no. 3, 201-214.

[AF] Alekseev A., Faddeev L., $\left(T^{*} G\right)_{t}$ : a toy model of conformal field theory, Comm. Math. Phys., 141, (1991) 413-422.

[AM] Alekseev A., Meinrenken M., The noncommutative Weil algebra, /math.DG 9903052, (1999).

[B] [B] Bernard, D., On the Wess-Zumino-Witten models on the torus, Nucl. Phys., B303, (1988) 77-93. 
[BBB] Babelon, O., Bernard, D., Billey, E.,A quasi-Hopf algebra interpretation of quantum 3-j and 6-j symbols and difference equations, Phys. Lett. B, 375 (1996) 89-97.

[BK-S] Bangoura M., Kosmann-Schwarzbach Y., Equations de Yang-Baxter dynamique classique et algébroides de Lie, C.R.Acad Sci. Paris, 327 (1998), no. 6, 541-546.

[BD] Belavin A.A, Drinfeld V.G, Triangle equations and simple Lie algebras, Soviet Sci. reviews, Sect C 4, 93-165. (1984).

[CP] Chari V., Pressley A., A guide to quantum groups, Cambridge University press, (1994).

[Dr] Drinfeld, V. On some open problems in quantum group theory, Lect. notes in Math, 1510, 1-8.

[E] Etingof, P.I., Quantum integrable systems and representations of Lie algebras, hep-th 9311132, J. Math. Phys. 36 (1995), no.6, 2636-2651.

[EF] Enriquez B., Felder G., Elliptic quantum groups $E_{\tau, \eta}\left(s l_{2}\right)$ and quasi-Hopf algebras, q-alg/9703018, Comm. Math. Phys., 195 (1998), no.3., 651-689.

[EFK] Etingof P., Frenkel I., Kirillov Jr. A., Lectures on representation theory and Knizhnik-Zamolodchikov equations AMS, (1998).

[EK] Etingof P., Kazhdan D., Quantization of Lie bialgebras I, Selecta Math. 2 (1996), $1-41$.

[EK1] Etingof, P.I., Kirillov, A.A., Jr, Macdonald's polynomials and representations of quantum groups, Math. Res. Let., 1 (3), (1994) 279-296.

[EK2] Etingof, P.I., Kirillov, A.A., Jr, Representation-theoretic proof of the inner product and symmetry identities for Macdonald's polynomials, Comp.Math., 102, (1996) 179-202.

[EK3] Etingof, P.I., Kirillov, A.A., Jr, On Cherednik-Macdonald-Mehta identities, math.QA 9712051, Electr. Res. Ann., (1998) 43-47.

[EK4] Etingof, P.I., Kirillov, A.A., Jr, On an affine analogue of of Jack and Macdonald polynomials, Duke Math.J., 78, (2) (1995) 229-256.

[ES1] Etingof P., Styrkas K., Algebraic integrability of Schrödinger operators and representations of Lie algebras, hep-th 9403135, Compositio. Math., 98, (1), (1995) 91-112.

[ES2] Etingof P., Styrkas K., Algebraic integrability of Macdonald operators and representations of quantum groups, q-alg 9603022, Compositio. Math. 114 (2) (1998), $125-152$.

[EV1] Etingof P., Varchenko A., Geometry and classification of solutions of the classical dynamical Yang-Baxter equation, Commun. Math. Phys, 192 77-120 (1998).

[EV2] Etingof P., Varchenko A., Solutions of the quantum dynamical Yang-Baxter equation and dynamical quantum groups, Commun. Math. Phys, 196 591-640 (1998).

[EV3] Etingof P., Varchenko A., Exchange dynamical quantum groups, preprint math.QA/9801135.

[EV4] Etingof P., Varchenko A., Traces of intertwiners for quantum groups and difference equations, I, preprint math.QA/9907181.

[Fad1] Faddeev L., On the exchange matrix of the WZNW model, Comm. Math. Phys., 132 (1990), 131-138. 
[F] Felder G., Conformal field theory and integrable systems associated to elliptic curves, Proceedings of the International Congress of Mathematicians, Zürich 1994, p.1247-1255, Birkhäuser, 1994; Elliptic quantum groups, preprint hepth/9412207, to appear in the Proceedings of the ICMP, Paris 1994.

[FR] Frenkel I., Reshetikhin N., Quantum affine algebras and holonomic difference equations, Commun. Math. Phys. 146 (1992), 1-60.

[FTV1] Felder G., Tarasov V., Varchenko A., Solutions of the elliptic QKZB equations and Bethe ansatz I, q-alg/9606005, in Topics in Singularity Theory, V.I.Arnold's 60th Anniversary Collection, Advances in the Mathematical Sciences -34, AMS Translations, Series 2, 180 (1997), 45-76.

[FTV2] Felder G., Tarasov V., Varchenko A., Monodromy of solutions of the elliptic quantum Knizhnik-Zamolodchikov-Bernard difference equations, q-alg/9705017, (1997), 1-26.

[FV1] Felder G., Varchenko A., On representations of the elliptic quantum group $E_{\tau, \eta}\left(s l_{2}\right)$, Commun. Math. Phys. 181 (1996), 746-762.

[FV2] Felder G., Varchenko A., Algebraic Bethe ansatz for the elliptic quantum group $E_{\tau, \eta}\left(s l_{2}\right)$, Nuclear Physics B 480 (1996) 485-503.

[FV3] Felder G., Varchenko A., Elliptic quantum groups and Ruijsenaars models, J. Statist. Phys. 89 (1997), no. 5-6, 963-980.

[FV4] Felder G., Varchenko A., Quantum KZB heat equation, modular transformations, and $G L(3, Z)$, I, math.QA/9809139, (1998).

[FV5] Felder G., Varchenko A., Resonance relations for solutions of the elliptic qKZB equations, fusion rules, and eigenvectors of transfer matrices of restricted interaction-round-a-face models, math.QA/9901111, (1999).

[FV6] Felder G., Varchenko A., Quantum KZB heat equation, modular transformations, and $G L(3, Z)$, II, math.QA 9907061, (1999).

[FV7] Felder G., Varchenko A., The elliptic gamma-function, and $S L_{3}(\mathbb{Z}) \ltimes \mathbb{Z}^{3}$, math.QA 9907061, (1999).

[Fr] Fronsdal C., Quasi-Hopf deformations of quantum groups, Lett. Math. Phys, 40, (1997) 117-134.

[GN] Gervais, J.-L., Neveu, A., Novel triangle relation and absense of tachyons in Liouville string field theory, Nucl. Phys. B, 238, (1984), 125-141.

[JKOS] Jimbo M., Konno H., Odake S., Shiraishi J., Quasi-Hopf twistors for elliptic quantum groups, q-alg 9712029, (1997).

[K1] Kirillov, A. Jr., Traces of intertwining operators and Macdonald polynomials, PhD thesis, q-alg 9503012, (1995).

[K2] Kirillov, A.Jr., On an inner product in modular tensor categories, J. Amer. Math. Soc, 9, (4), (1996) 1135-1169.

[K3] Kirillov, A. Jr, On an inner product in modular tensor categories, II, Adv.Theor.Math.Phys, 2, (1998) 155-180.

[Lu] Lu J.H., Hopf algebroids and quantum groupoids, Inter. J. Math., 7 (1), (1996), 47-70.

[LX] Liu, Z.-J., Xu, P., Dirac structures and dynamical r-matrices, math.DG/9903119, (1999).

[M] Mackenzie K, Lie groupoids and Lie algebroids in differential geometry, Cambridge Univ. Press, 1997.

[Ma] Macdonald, I.G., A new class of symmetric functions, Publ. I.R.M.A. Strasbourg, 372/S-20, Actes 20 Seminaire Lotharingien (1988) 131-171. 
[MV] Mukhin E., Varchenko A., Solutions of the $q K Z B$ equations in tensor products of finite dimensional modules over the elliptic quantum group $E_{\tau, \eta} s l_{2}, 9712056$, (1997).

[S] Schiffmann O, On classification of dynamical r-matrices, Math. Res. Letters, 5, 13-30 (1998).

[TV1] Tarasov T., Varchenko A., Geometry of q-Hypergeometric functions as a bridge between Yangians and Quantum Affine Algebras, Inv.Math., 128, (1997), 501-588.

[TV2] Tarasov V., Varchenko A., Geometry of q-Hypergeometric Functions, Quantum Affine Algebras and Elliptic Quantum Groups, q-alg/9703044, Asterisque, 246, (1997), pages 1-135.

[W] Weinstein A., Coisotropic calculus and Poisson groupoids J. Math. Soc. Japan 40, 705-727, (1988).

[Xu1] Xu, P., Quantum groupoids associated to universal dynamical R-matrices, C.R.Acad. Sci. Paris, Serie I, 328, (1999) 327-332.

[Xu2] Xu, P., Quantum groupoids, math.QA 9905192, (1999). 